\title{
Sox9 and Rbpj differentially regulate endothelial to mesenchymal transition and wound scarring in murine endovascular progenitors
}

\author{
Jilai Zhao (1) 1,6, Jatin Patel 1,2,6, Simranpreet Kaur ${ }^{1}$, Seen-Ling Sim¹, Ho Yi Wong ${ }^{1}$, Cassandra Styke (1) 1, \\ Isabella Hogan ${ }^{1}$, Sam Kahler (1) 1, Hamish Hamilton ${ }^{1}$, Racheal Wadlow ${ }^{1}$, James Dight ${ }^{1}$, Ghazaleh Hashemi (i) ${ }^{1}$, \\ Laura Sormani', Edwige Roy (iD ${ }^{1}$, Mervin C. Yoder (iD ${ }^{3}$, Mathias Francois ${ }^{4,5}$ \& Kiarash Khosrotehrani (i) ${ }^{1 凶}$
}

Endothelial to mesenchymal transition (EndMT) is a leading cause of fibrosis and disease, however its mechanism has yet to be elucidated. The endothelium possesses a profound regenerative capacity to adapt and reorganize that is attributed to a population of vesselresident endovascular progenitors (EVP) governing an endothelial hierarchy. Here, using fate analysis, we show that two transcription factors SOX9 and RBPJ specifically affect the murine EVP numbers and regulate lineage specification. Conditional knock-out of Sox 9 from the vasculature (Sox fl/fl/Cdh5-Cre $^{E R}$ RosaYFP) depletes EVP while enhancing Rbpj expression and canonical Notch signalling. Additionally, skin wound analysis from Sox9 conditional knock-out mice demonstrates a significant reduction in pathological EndMT resulting in reduced scar area. The converse is observed with Rbpj conditionally knocked-out from the murine vasculature (Rbpj/ffl/Cdh5-CreER RosaYFP) or inhibition of Notch signaling in human endothelial colony forming cells, resulting in enhanced Sox9 and EndMT related gene (Snail, Slug, Twist1, Twist2, TGF- $\beta$ ) expression. Similarly, increased endothelial hedgehog signaling (Ptch $f^{f / f l} /$ Cdh5-CreER RosaYFP), that upregulates the expression of Sox9 in cells undergoing pathological EndMT, also results in excess fibrosis. Endothelial cells transitioning to a mesenchymal fate express increased Sox9, reduced Rbpj and enhanced EndMT. Importantly, using topical administration of siRNA against Sox 9 on skin wounds can substantially reduce scar area by blocking pathological EndMT. Overall, here we report distinct fates of EVPs according to the relative expression of Rbpj or Notch signalling and Sox9, highlighting their potential plasticity and opening exciting avenues for more effective therapies in fibrotic diseases.

\footnotetext{
${ }^{1}$ The University of Queensland Diamantina Institute, The University of Queensland, Woolloongabba, QLD, Australia. ${ }^{2}$ Centre for Ageing Research Program, Queensland University of Technology, Woolloongabba, QLD, Australia. ${ }^{3}$ Indiana Center for Regenerative Medicine and Engineering, Indianapolis, IN, USA. ${ }^{4}$ The David Richmond Laboratory for Cardiovascular Development: Gene Regulation and Editing Program, The Centenary Institute, Camperdown, NSW, Australia. ${ }^{5}$ The School of Life and Environmental Sciences, Faculty of Science, The University of Sydney, Camperdown, NSW, Australia. ${ }^{6}$ These authors contributed equally: Jilai Zhao, Jatin Patel. ${ }^{凶}$ email: k.khosrotehrani@uq.edu.au
} 
$\mathrm{D}$ uring adult life the endothelial layer that lines the lumen of the vascular system is heterogeneous in phenotype and retains the capacity to drive tissue regeneration and repair with responsive adaptations to both physiological and pathological conditions $^{1,2}$. However, mechanisms that drive endothelial activity and plasticity, in particular those that contribute to fibrotic disease associated with adult wound healing through a process of endothelial to mesenchymal transition (EndMT) remain to be elucidated ${ }^{3,4}$. In prenatal development, EndMT is essential in endocardial cushion formation, cardiac fibroblasts and smooth muscle cell generation ${ }^{5,6}$. By contrast, in the adult vascular network, EndMT heavily influences the pathogenesis of several fibrotic diseases and is an essential process during wound healing ${ }^{7}$. In the cardiac setting, Zeisberg et al., (2007) demonstrated that EndMT played a significant role in the formation of excessive cardiac fibrosis, in situations of cardiac injury and subsequent repair ${ }^{8}$. Endothelial cells (ECs) adopted a fibroblast-like fate, becoming more proliferative, thrombogenic and expressed large amounts of extracellular matrix (ECM) proteins, resulting in cardiac fibrosis and driving disease progression. Similarly, during cutaneous wound healing, after a peak in neo vessel formation, ECs undergo EndMT and contribute to scarring9. These examples highlight the importance of endothelial plasticity and cell fate decision during health and disease. During wound healing, the endothelium contributes to the expansion of the vasculature by adopting endothelial phenotypes and later, transitioning towards a mesenchymal phenotype, it contributes to fibrosis using yet unclear mechanisms.

Recently, we and others demonstrated that a tissue resident endovascular progenitor (EVP) exists throughout the circulatory system, which governs an endothelial hierarchy ${ }^{10-12}$ that leads to de novo blood vessel formation. During homoeostasis and disease, quiescent EVPs give rise to rapidly proliferating transit-amplifying cell, which then subsequently adopt a mature endothelial cell phenotype connected to the circulation ${ }^{10,13-15}$. In particular, during skin wound healing, EVPs are found in the centre of the granulation tissue at day 1 (D1) and give rise to transit amplifying (TA) and differentiated endothelial (D) cells that can only be visualised from D3 post wounding ${ }^{10}$. The vessel formation peaks at D5 and then regresses giving room to a mesenchymal transition of ECs contributing to the scar'. Gene expression studies have identified two transcription factors (TFs), Sox9 and Rbpj, as being key markers distinguishing EVPs from other endothelial populations ${ }^{14}$. Sox 9 has been reported in other stem cell compartments, in particular, in hair follicle stem cells but also in controlling chondrocytes lineage specification of mesenchymal cells as well as neuronal stem cells development ${ }^{16-19}$. Importantly, high expression of Sox9 has been implicated in driving/exacerbating fibrotic diseas $\mathrm{e}^{20,21}$. On the other hand, canonical Notch signalling through activity of its main effector transcription factor Rbpj is known to guide endothelial function and specification ${ }^{22}$. In other contexts, Rbpj and Sox9 have been shown to have antagonistic roles, whereby excess expression of Rbpj resulted in depleting Sox 9 expression in chondrocytes driving chondrodysplasia ${ }^{23}$. Conversely, we have previously shown that when $R b p j$ is conditionally deleted in the endothelium, this resulted in accelerated EndMT, with excessive fibrosis and scar tissue formation observed in a skin excisional wound healing scenario 9 .

These lines of evidence point towards a genetic interaction between $S o x 9$ and $R b p j$ and prompted us to investigate a functional role of Sox9 specifically in the endothelium. We asked how these TFs orchestrate cell fate decision at the crossroad between vascular endothelium and mesenchymal transition. In this study we report that conditional deletion of Sox 9 from the endothelium correlates with increased $R b p j$ expression and a significant reduction in EndMT and therefore fibrosis. Alternatively, indirect overexpression of Sox9 through loss of Rbpj or activation of hedgehog $(\mathrm{HH})$ signalling in the endothelium produces opposite effects.
Using a pre-clinical model of wound healing, we establish the proof of principle that Sox9 gene knock-down with siRNA applied to wounds results in abrogation of EndMT and reduces the scar area, providing potential therapeutic avenues in blocking fibrosis.

\section{Results}

Expression of Sox9 in the mouse and human endothelium. SOX9 protein expression has been associated with many stem cell types but hardly associated with the endothelium. In previous independent studies from our group, we have reported Sox 9 mRNA expression through bulk or single cell RNA sequencing in murine EVPs isolated from either the aorta or the tumour endothelium ${ }^{10,13}$. Additionally, Sox9 mRNA expression has also been reported in coronary arteries and the aorta as observed in the Genotype-Tissue Expression (GTEx) project as well as in the Atlas of single cell RNA sequencing of the murine endothelium ${ }^{24}$. To explore and confirm the expression of the transcription factor SOX9 in the endothelium, a variety of scenarios were assessed. Thoracic and abdominal aortic tissue was isolated from wild-type C57Bl/6 mice before being processed for en face SOX9 immunofluorescence whole-mount staining. Stochastic clustering of SOX9 positive nuclei (labelled yellow) was observed throughout the aorta within cells harbouring the pan endothelial marker CD31(labelled red) (Fig. 1a). Similarly, in whole mounted normal skin, rare ECs expressed SOX9 (Fig. 1b). Additionally, we have tested the specificity of the SOX9 antibody and shown clear positive and specific staining of the nuclei of hair follicle bulge stem cells in skin (Supplementary Fig. 1a). Moreover, in a Sox9 knock-out situation no specific staining could be observed (see below). We also observed that the SOX9 staining is localised only to the endothelial layer and not in the underlying vascular smooth muscle layers (Supplementary Fig. 1b).

To better understand the frequency of SOX9 expression, aortic cells expressing VE-Cadherin and CD34 but devoid of hematopoietic markers (Lin-VE-Cad+CD34+, Fig. 1c) were FACS sorted and separated into individual EVP (CD31 $1^{\text {low }}$ VEGFR2 $\left.{ }^{\text {low }}\right)$ and mature differentiated endothelial $\left(\mathrm{D}, \mathrm{CD} 31^{\mathrm{Hi}}, \mathrm{VEGFR} 2 \mathrm{Hi}\right)$ cells that were then cytospun onto slides to be stained for SOX9, ERG and RBPJ. As expected, only EVP cells stained positively for SOX9 as opposed to D cells. Importantly however, both EVP and D cells stained positively for the endothelial specific transcription factor marker ERG, demonstrating that SOX9 is indeed expressed in the endothelium (Fig. 1c). Moreover, RBPJ was found to be expressed in both EVP and D cells. EVPs that expressed RBPJ could be found to express SOX9 as well. Quantification of sorted aortic cells could show that only about a third of EVPs expressed SOX9, whereas most expressed RBPJ (Supplementary Fig. 1c and Fig. 1c). Finally, both RBPJ and SOX9 were significantly more frequently expressed at protein level in EVPs compared to D cells (SOX9 vs D cells $p<0.001$; RBPJ vs D cells $p<0.001$; Fig. 1c).

Previously, we have reported the presence of EVP cells in excisional wound granulation tissue at $\mathrm{D} 1^{10}$. To confirm the expression of SOX9 in these cells, we next performed SOX9 immunostaining in wounds at this time-point ${ }^{10}$. Here we used a vascular lineage tracing mouse model, Cdh5-Cre ${ }^{E R}$ RosaYFP, provided 10 days of tamoxifen via intraperitoneal injection (IP) to label the entire vasculature with YFP before full thickness excisional wounds were created on the back of mice. Colabelling of the lineage tracing mark YFP and CD31 in a variety of tissues collected immediately after tamoxifen injection demonstrated a very strong overlap in signal demonstrating the specificity of endothelial lineage labelling upon Cre induction (Supplementary Fig. 2a). Upon tamoxifen induction and $24 \mathrm{~h}$ after wounding, most YFP + cells in the centre of the wound were individual EVP cells with SOX9 positive nuclei further 
a

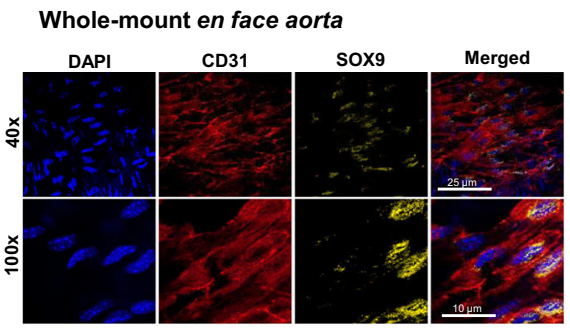

b
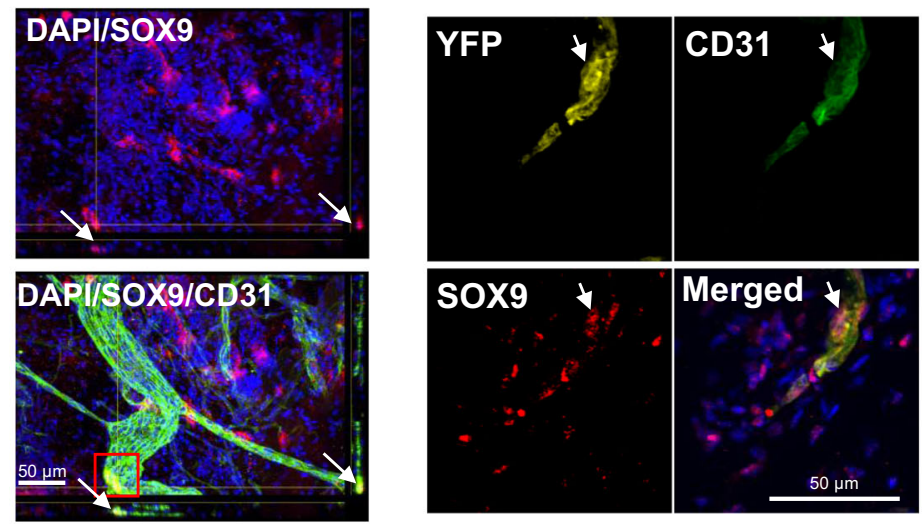

c
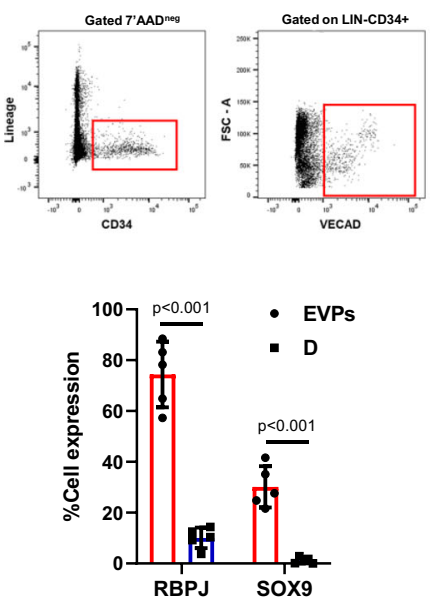

d
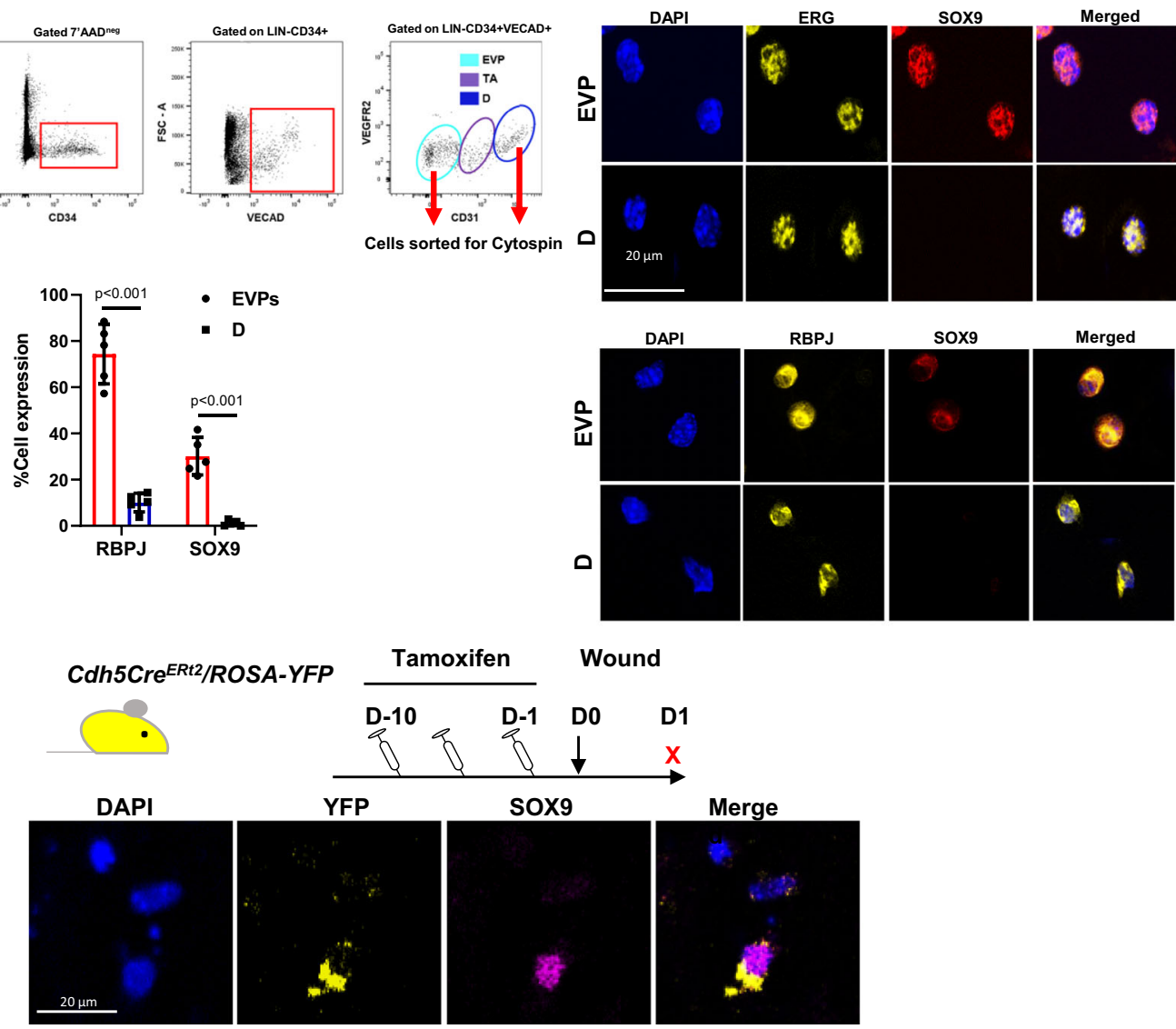

Merged

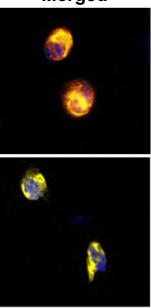

Wound

D0 D1
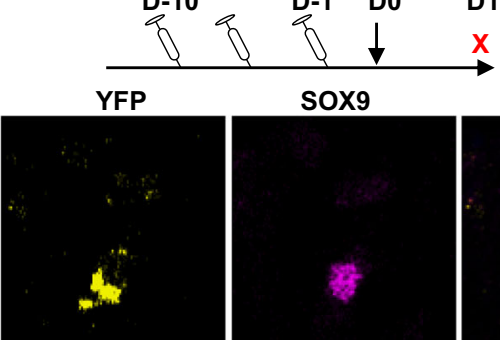

sox 9

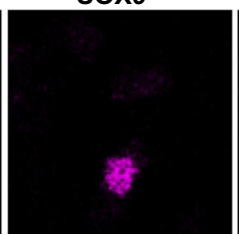

Merge

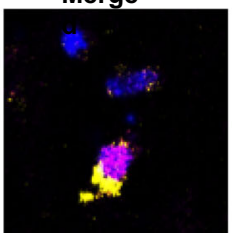

confirming the expression of SOX9 in YFP + ECs in a pathological scenario (Fig. 1d). Moreover, the use of a Sox9Cre/ER reporter mouse showed Cre expression in the aortic and wound endothelium, demonstrating the expression of Sox9 at the RNA level in these tissues (Supplementary Fig. 2b).

These data in addition to our previous reports on mRNA expression provide strong evidence to suggest that the expression of SOX9 is observed in ECs and is restricted to a specific EVP population within the endothelium.

SOX9 expression is essential for EVP maintenance and quiescence. To assess the molecular function of SOX9 in the endothelium we generated a Sox9 endothelial specific conditional 
Fig. 1 Expression of Sox 9 in the mouse and human endothelium. a Whole-mount aorta staining demonstrating SOX9 nuclear staining among endothelial cells (Sox9 in yellow, CD31 in red). b Whole-mount skin staining demonstrating SOX9 expression in dermal vasculature (white arrow; SOX9 positive endothelial cells), immunofluorescent staining of skin sections collected from the Cdh5-Cre ER RosaYFP mice displays nuclear SOX9 expression in a YFP + endothelial cell. c FACS sorting gating strategy, endovascular progenitor (EVPs) and mature differentiated endothelial (D) cells were FACS sorted and cytospun for staining. FACS sorted EVP showing positive co-staining for ERG, and SOX9 as well as RBPJ with SOX9. D cells are only positive for ERG and RBPJ but not SOX9. Quantification demonstrates a significantly larger percentage of EVPs are positive for RBPJ and SOX9 compared to D cells ( ${ }^{\star \star *} p<$ $0.005 ; n=3$; cell sorted from three biologically independent animals; mean \pm SD; $p$ value was calculated by two-way ANOVA with multiple comparison of row mean) d Immunofluorescence staining of day 1 (D1) wound sections collected from Cdh5-Cre ${ }^{E R}$ RosaYFP mice show that YFP + yellow cells within the centre are SOX9 positive.

knock-out that we termed a Sox9eKO (Sox9fl/fl/Cdh5-Cre $e^{E R}$ RosaYFP). Both Sox $9^{e W T}$ (Sox $9^{+/+} / C d h 5-C r e^{E R}$ RosaYFP) and Sox $9^{e K O}$ were provided tamoxifen injection IP for 10 consecutive days at adult age (10-12 weeks of age). The mice were culled, and the aortic tissue harvested for assessment of the endothelium (Fig. 2a). Upon tamoxifen injection, Sox 9 mRNA could not be detected in Sox $9^{e K O}$ ECs. Similarly, SOX9 immunofluorescence failed to detect any remaining protein in the aorta from mice with conditionally deleted Sox9 (Supplementary Fig. 3a). Fluorescence minus one (FMO) was used to determine negative and positive cut-offs for flow cytometry analysis (Supplementary Fig. 2b). Sox $9^{e W T}$ aortas displayed the endothelial hierarchy previously described $^{10}$ among Lin-YFP + CD34 + cells. All three populations (EVP, TA and D) were observable (Fig. 2b). However, in the Sox $9^{e K O}$ mice, a greater than two-fold significant depletion in the number of EVP cells was observed. This coincided with a significant increase in the $\mathrm{D}$ population numbers $(* * p<0.01$; Fig. 2c). Of importance, cell cycle regulators such as Il33, $p 16, p 21$ and $p 57$ showed over fourfold reduction in mRNA expression in EVPs isolated from Sox $9^{e K O}$ mice suggesting a role for Sox 9 in maintaining EVPs in quiescence $(* * * p<0.001 \text {; Fig. } 2 \mathrm{~d})^{25}$. We next addressed the functional importance of SOX9 in the endothelium in disease.

Loss of Sox9 in the endothelium significantly impairs EndMT and reduces scarring during wound healing. Given the importance of EVPs in neo-vessel formation during wound healing, we used excisional wounds to evaluate the functional importance of SOX9 expression in EVPs. Here Sox $9^{e W T}$ and Sox $9^{e K O}$ mice were injected IP for 10 days with tamoxifen before undergoing full thickness large skin excisional $(1.5 \mathrm{~cm} \times 1.5 \mathrm{~cm})$ wounds on their dorsal skin allowing us to evaluate wound healing and scar tissue formation (Fig. 3a). Large excisional wounds were chosen to allow a direct macroscopic measurement of the scar area and results were confirmed in small $6 \mathrm{~mm}$ excisional wounds through microscopic examination. The wounds from each group were monitored every 3 days and wound area was measured (Fig. 3b, c; $n=8$ per group). Intriguingly, the Sox $9^{e K O}$ mice displayed significantly faster wound healing capacity compared to the Sox $9^{e W T}$ controls from D12 onwards $\left(* p<0.05\right.$; ${ }^{* *} p<0.01$ vs Sox $\left.9^{e W T}\right)$.

The scar quality was then assessed in $6 \mathrm{~mm}$ full thickness punch biopsy wounds on the dorsum of Sox $9^{e W T}$ and Sox $9^{e K O}$ mice. We had observations of reduced scarring using Sirius red staining upon Sox9 deletion in the endothelium (Fig. 3e). Wounds were also harvested at day 5 (D5) and 7 (D7) to evaluate EndMT (Supplementary Figs. 4, 5 and Fig. 4a). We first assessed if changes in the endothelial fate could be observed via flow cytometry. We established a new gating strategy to allow tracking of all populations labelled with YFP upon lineage tracing by gating on live Lin-YFP + cells (Supplementary Fig. 4). Gated cells were then distinguished based on their level of CD34 and CD31 surface expression. Comparison between the two gating strategies allowed the identification of EVP and D cells as well as
CD31 + cells that did not express CD34 extending from the D population. This new gating strategy allowed the visualisation of a CD34-CD31-population that expressed YFP. Further staining showed that the latter population expressed mesenchymal markers such as CD26 or PDGFRa at higher levels compared to endothelial populations. Moreover, upon sorting and immunostaining, these cells expressed aSMA and not CD31, in contrast to D cells (Supplementary Fig. 4). This population was therefore labelled mesenchymal (M) and could be identified in the aorta but at relatively higher levels in the normal skin.

Using this new gating strategy, we next performed flow cytometry evaluation of skin wounds at D5 and D7 (Supplementary Fig. 5a, b). As reported previously by us and others, the total number of ECs (Lin- and VE-cadherin + cells) reduced between D5 and D7 reflecting the peak in angiogenesis often reported in D5. Sox $9^{e K O}$ animals had equal number of ECs in D5 but higher numbers in D7 compared to controls. We observed a significant reduction in the number of EVP cells within the granulation tissue of wounds at D5 in Sox $9^{e K O}$ in comparison to controls $(p<$ 0.001 vs So $x 9^{e W T}$; Supplementary Fig. 5b) although this difference was not significant in D7 given the previously reported reduction of EVP cells in wounds at $\mathrm{D} 7^{10}$. This resulted in a relative increase in $\mathrm{D}$ cells but no significant change in the absolute number of D cells at both D5 and D7. Next, using the new gating strategy, we could evaluate EndMT through quantification of the $M$ population. The $M$ population was deemed to exemplify EndMT as it originated from ECs given its YFP lineage label and had subsequently lost CD34 and CD31 and gained mesenchymal markers such as CD26 26 or PDGFRa. Although $M$ cells did not differ in numbers at D5, they were significantly reduced at D7, a time-point past peak angiogenesis when EndMT starts to occur as we have reported previously 9 $\left(p=0.024\right.$ vs Sox $9^{e W T}$, Supplementary Fig. 5b).

Next we evaluated EndMT in tissue sections using three classical mesenchymal markers, $\alpha$-SMA, FSP1 and SLUG, alongside endothelial markers YFP and CD31 at D79(Fig. 4b). The number of vessels and individual cells that co-expressed YFP $+\alpha$ $\mathrm{SMA}+\mathrm{CD} 31+$ was significantly reduced in Sox $9^{e K O}$ mice compared to controls $\left(p<0.001\right.$ vs Sox $9^{e W T}$ Fig. $\left.4 b, c\right)$. In this assessment, we took extreme care to avoid counting mature vessels harbouring a-SMA + pericyte on their periphery and only true overlap of the triple staining was taken into account. Additionally, the number of vessels that had entirely lost endothelial phenotype (YFP $+a-S M A+C D 31-)$ was also significantly reduced in Sox $9^{e K O}$ mice compared to controls $(p<$ 0.001 vs Sox $9^{e W T}$ Fig. 4c). Of importance, the latter category included individual dermal cells detached from the structure of classical blood vessels (Supplementary Fig. 5c). We next assessed a key EndMT transcription factor $\operatorname{SLUG}^{27,28}$ as well as the fibroblast marker FSP1 (S100A4) expression and identified again a significant reduction in both vessels and cell counts in YFP + structures that had lost CD31 expression $\left(p<0.001\right.$ vs Sox $9^{e W T}$; Fig. 4c). Overall, these findings suggest that the deletion of Sox9 specifically from the endothelium reduces the likelihood of 
a

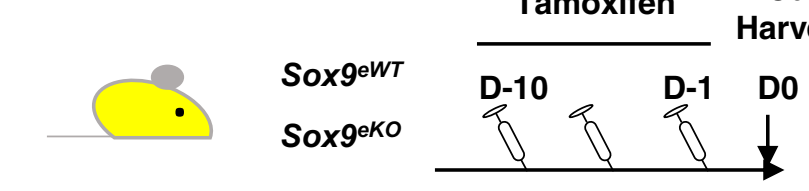

Cull Mice, Harvest Aorta
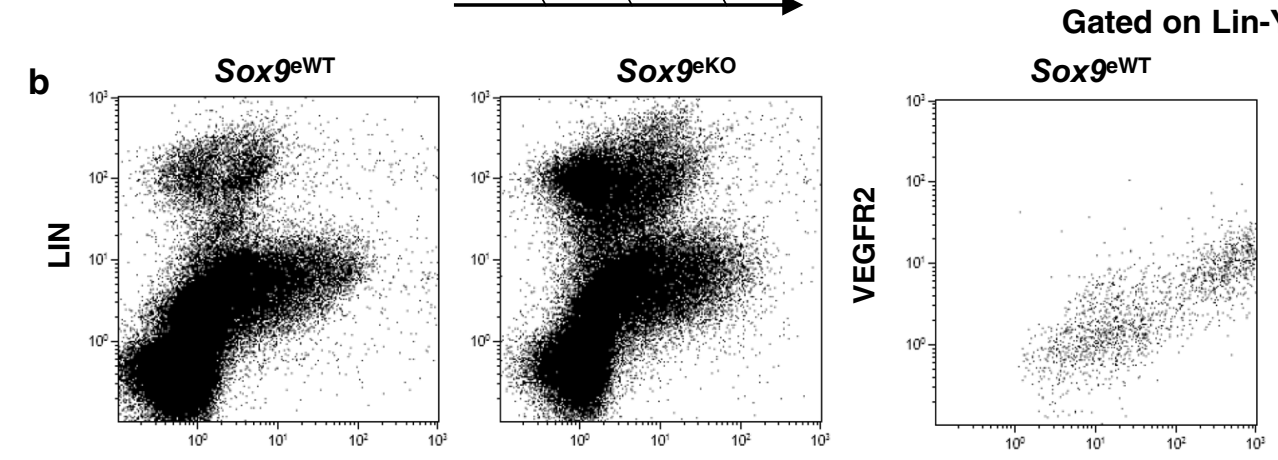

Gated on Lin-YFP+CD34+
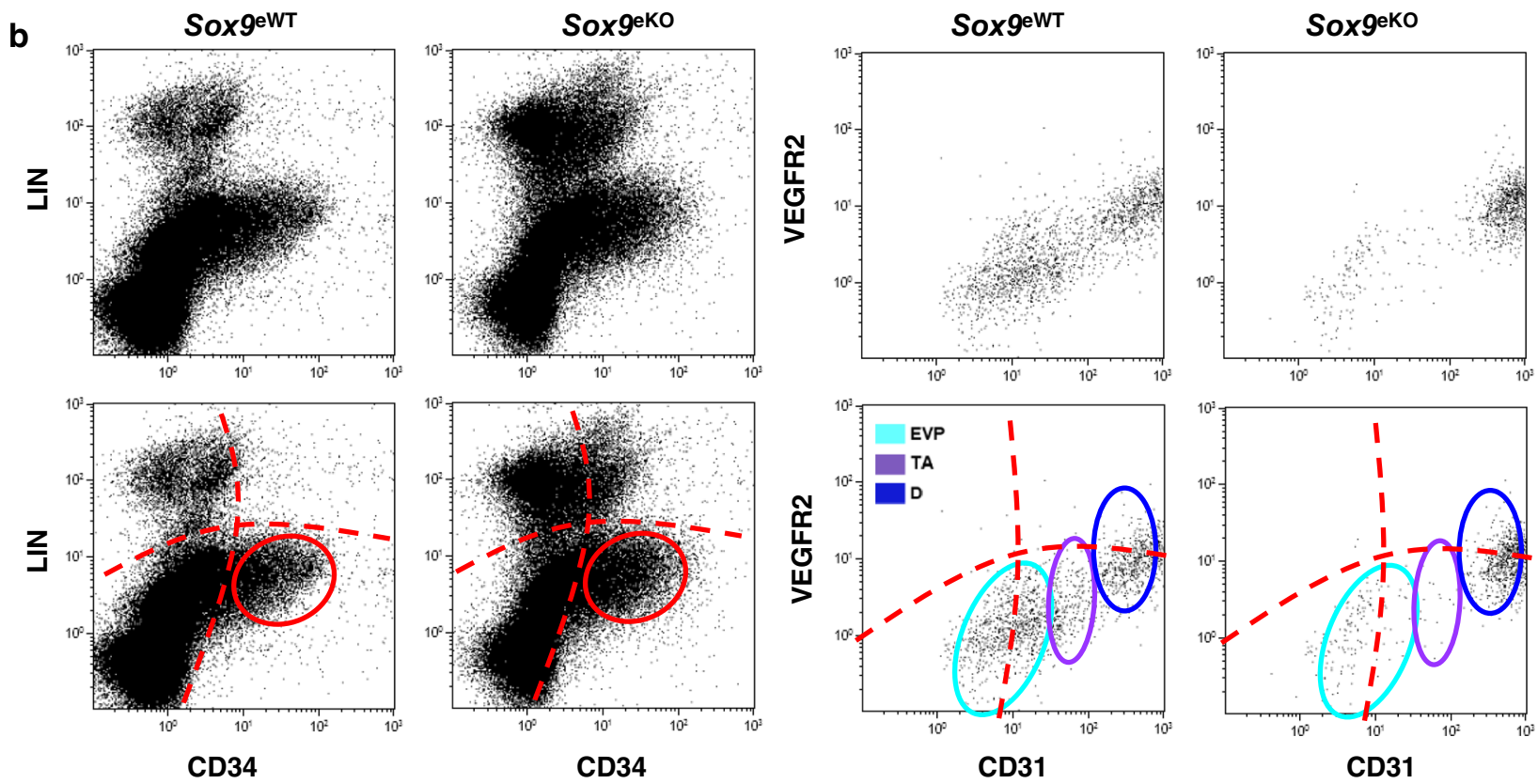

C

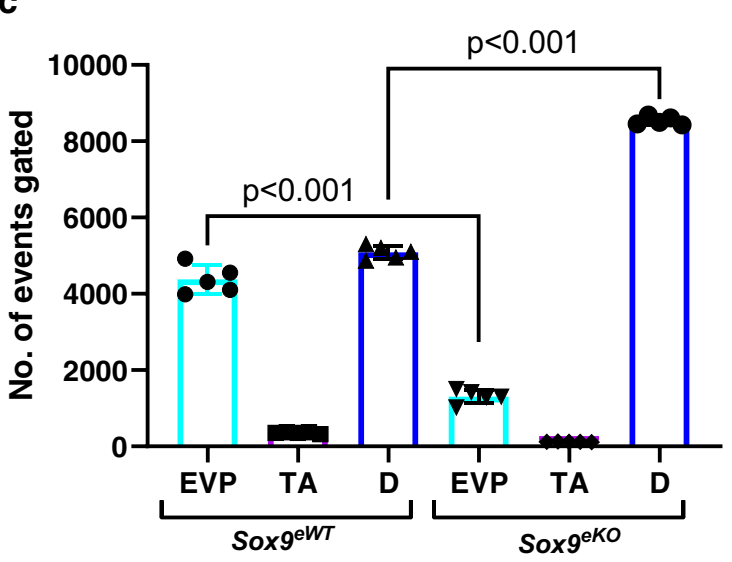

d

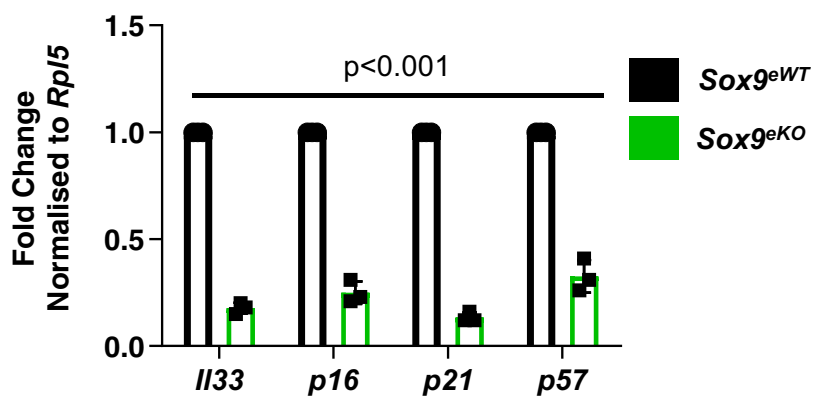

Fig. 2 SOX9 expression is essential for endovascular progenitor clonogenicity and quiescence. a Schematic diagram demonstrating experimental set up using conditional vascular specific Sox9 knockout mice (Sox9f//fl/Cdh5-Cre ER RosaYFP - SoxgeKO) and sample collection 12 weeks post-induction. b Flow cytometry plots showing the aortic endothelium harbour a distinct CD34 positive, lineage (Lin) negative population (red gate) that is entirely YFP positive, as determine by fluorescence minus one (FMO) analysis. Three distinct populations were observed based on CD31 and VEGFR2 expression (from left to right endovascular progenitor EVP; transit amplifying TA; differentiated D) showing the endothelial hierarchy and changes between wild-type controls $\left(\right.$ Sox $9^{e W T}$ ) and Sox $9^{e K O}$ mice after 10 days tamoxifen. c Number of EVP is significantly reduced in Sox $9^{e K O}$ ( $p<0.001$ vs Sox $9^{e W T} ; n=3$ biologically independent animals; mean \pm SD). $\mathbf{d}$ qPCR analysis of genes associated with endothelial quiescence in EVP $\left(^{\star \star \star} p<0.001\right.$ vs Sox ${ }^{\text {eWT; }} n=3$; mean \pm SD).

EndMT, thus resulting in reduced fibrosis and scarring in skin wounds.

Sox9 deletion results in increased Notch signalling with reduced expression of TGF $\beta$ and EndMT target genes. To understand the gene expression consequences of SOX9 deletion in the endothelium, aortic tissue from Sox $9^{e W T}$ and Sox $9^{e K O}$ mice was harvested and EVP were FACS sorted and processed for analysis by quantitative polymerase chain reaction (qPCR). Key notch signalling effector, $R b p j$, increased by over 4.5 -fold in Sox $9^{e K O}$ compared to controls and was associated with an upregulation of expression in canonical Notch signalling target genes such as Hey1 and Hes1. Classical EndMT genes such as TGF- $\beta$, Snail, Slug and Twist were all downregulated by over 4-fold, suggesting the upstream importance of Sox 9 in this pathway classically attributed to TGF $\beta$ signalling ${ }^{28,29}$ ( $p<0.002$ vs Sox9 ${ }^{e W T}$; Fig. $4 d$, e). The changes in gene expression further support that loss of Sox9 results in decrease in EndMT and its associated genes. It also resulted in an increase in $R b p j$ expression possibly driving the maintenance of an endothelial fate, blocking EndMT. Indeed, we previously reported that loss of $\mathrm{Rbpj}$ in the endothelium resulted in increased EndMT and scarring in the context of wound healing? 
a Tamoxifen Wound Healing
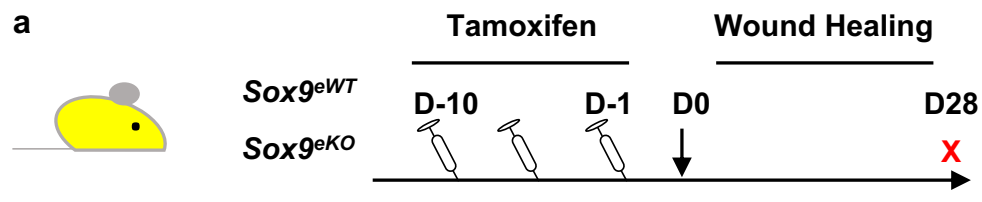

b
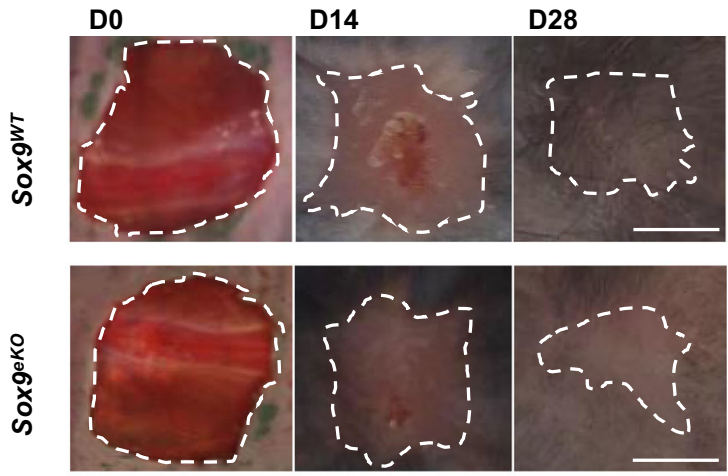

C

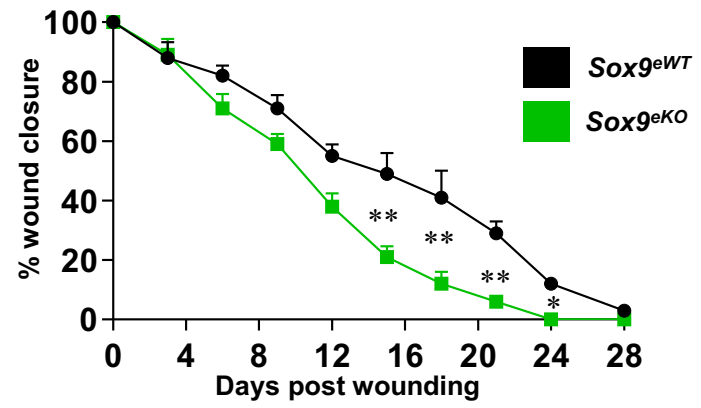

d
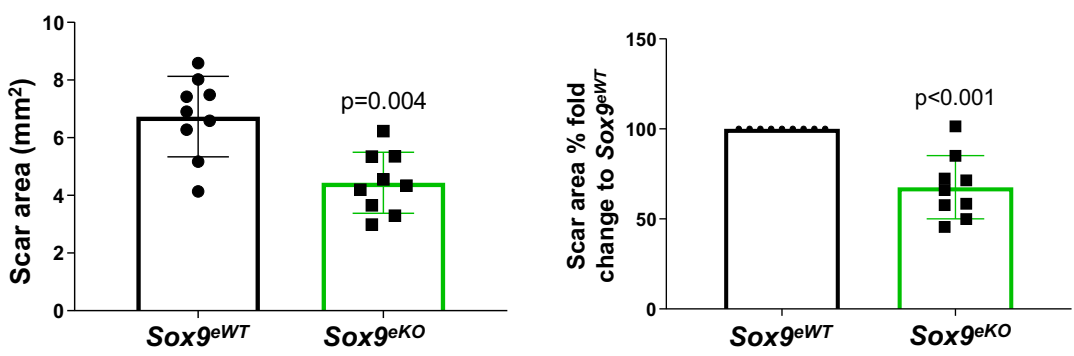

e
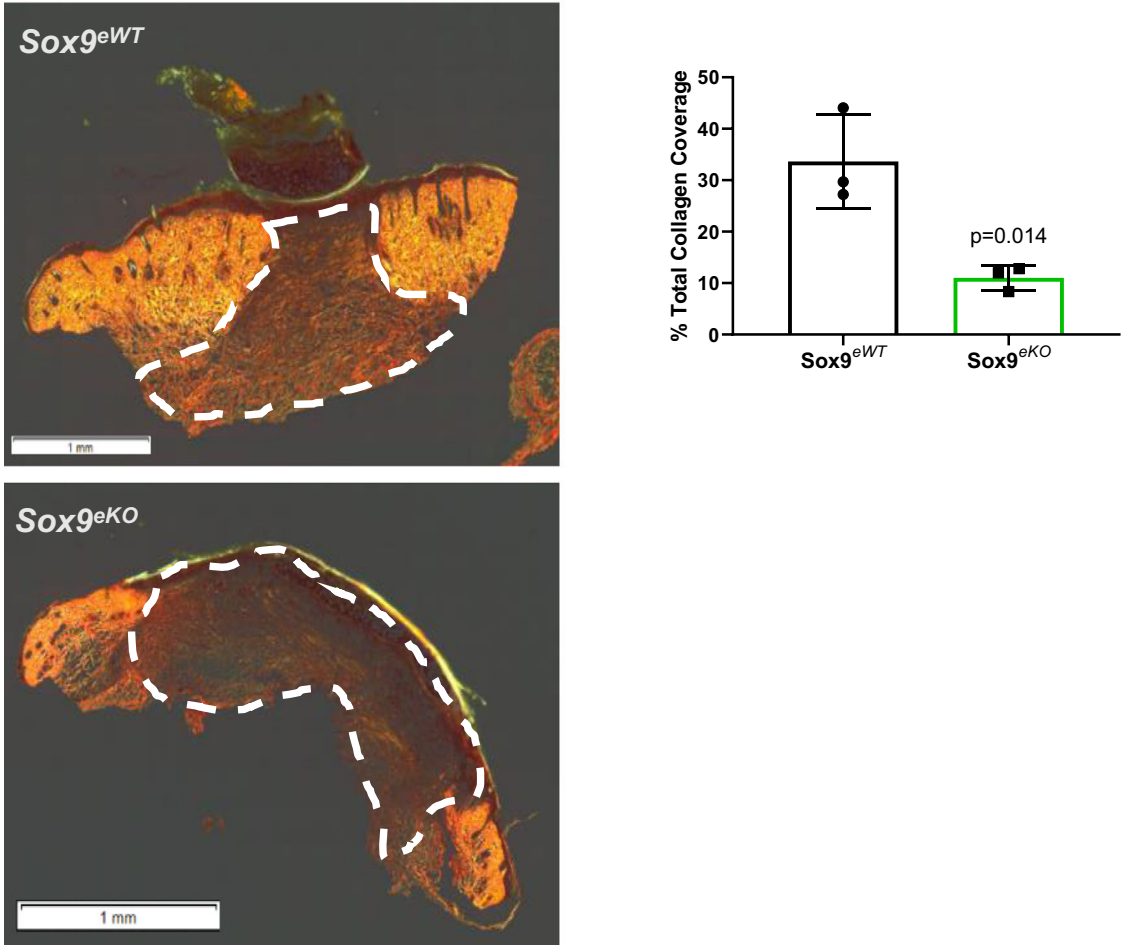

Fig. 3 Loss of Sox 9 in the endothelium significantly impairs EndMT and reduces scarring during wound healing. a Schematic diagram demonstrating experimental set up using conditional vascular specific Sox9 knockout mice (Sox $9^{f / f l} /\left(\mathrm{Cdh} 5-C r e^{E R}\right.$ RosaYFP - Sox $9^{e K O}$ ). b Digital photography images showing macroscopic wound healing over the course of 28 days (D28) post wounding between wild-type controls (Sox9eWT) and SoxgeKO. Scale bar $=5 \mathrm{~mm}$. c Skin wound healing rate as a percentage of initial wounded area was significantly increased in Sox 9 eKO compared to Sox $9^{\mathrm{eWT}}\left({ }^{\star} p<0.05\right.$; ${ }^{* \star} p<0.01$ vs Sox ${ }^{{ }^{e W W}}$; $n=8$ biologically independent animals; mean $\pm S D$; $p$ value was calculated by two-tailed unpaired $t$ test). $\mathbf{d}$ After 28 days, a significant decrease in macroscopic scar area was observed in Sox 9 eKO mice compared to Sox ${ }^{e W T}$ ( $p=0.004 ; n=8$ biologically independent animals; mean \pm SD; $p$ value was calculated by two-tailed unpaired $t$ test) e Sirius red collagen staining of both $6 \mathrm{~mm}$ and large wounds demonstrates a significant decrease in total collage coverage within the granulation tissue in SoxgeKO compared to SoxgeWT ( $p=0.0014$ vs SoxgeWT; $n=3$ biologically independent animals; mean \pm SD; $p$ value was calculated by two-tailed unpaired $t$ test). 
a

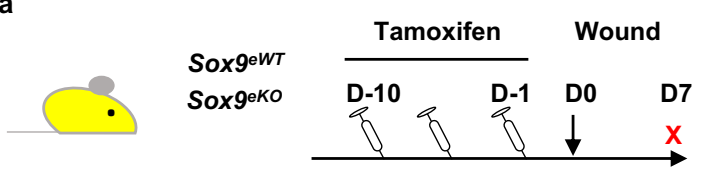

b
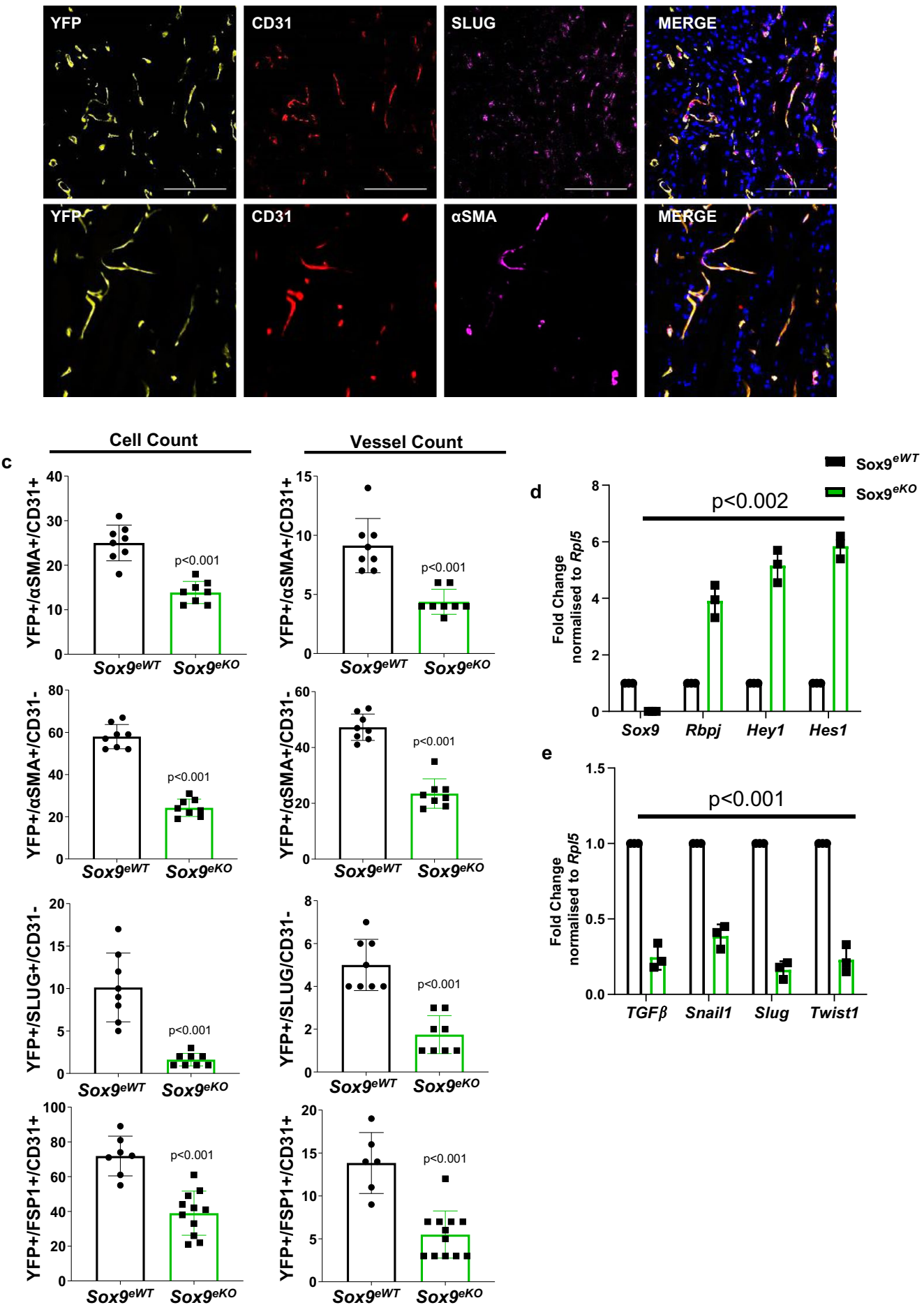

Fig. 4 Quantification of endothelial to mesenchymal transition (EndMT) upon loss of SOX9. a Schematic diagram demonstrating experimental set up using conditional vascular specific Sox 9 knockout mice (Sox $9^{f / / f l} / C d h 5-C r e^{E R} R o s a Y F P-S o x 9^{e K O}$ ). b Examples of immunofluorescence staining of skin wound sections at day 7 (D7) with endothelial markers CD31, lineage marker YFP and EndMT markers SLUG or $\alpha$ SMA. Scale bar $=50 \mu$ m. c Quantification of EndMT showing overlap of YFP + cells with either endothelial and mesenchymal markers such as SLUG, $\alpha S M A$ and FSP1 (** $p<0.01$ vs Sox 9 eWT; $n=5$ biologically independent animals; mean \pm SD; $p$ value was calculated by two-tailed unpaired $t$ test). $\mathbf{d}, \mathbf{e}$ qPCR analysis of key endothelial and EndMT genes in EVP from Sox9 9 KO compared to Sox $9{ }^{e W T}$ ( ${ }^{\star \star} p<0.01$; ${ }^{\star \star \star} p<0.001$ vs Sox 9 eWT; $n=3$; cells were sorted from three groups of five biologically independent animals; mean $\pm \mathrm{SD}$; $p$ value was calculated by two-way ANOVA with multiple comparison of row mean). 
a

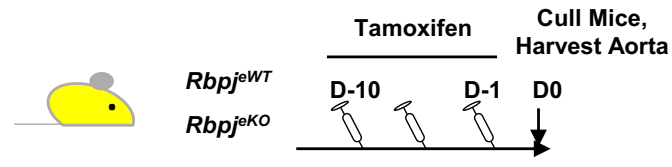

Sorted YFP+LIN ${ }^{\text {negCD34+ }}$
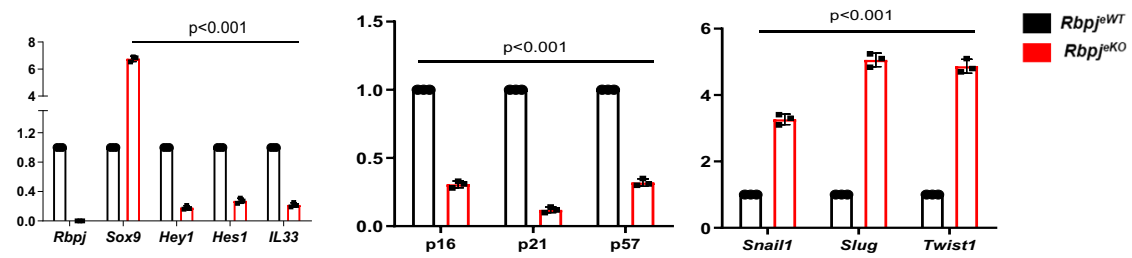

b

SoxgewT

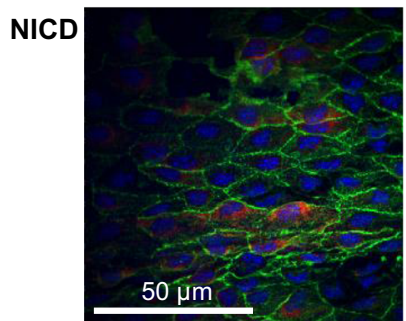

Sox 9 eKo

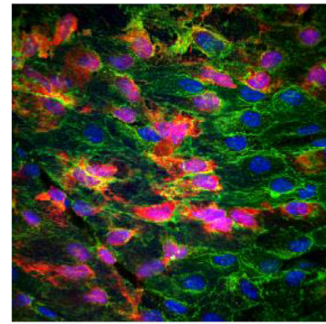

SOX9 $R{ }^{2 b j e W T}$
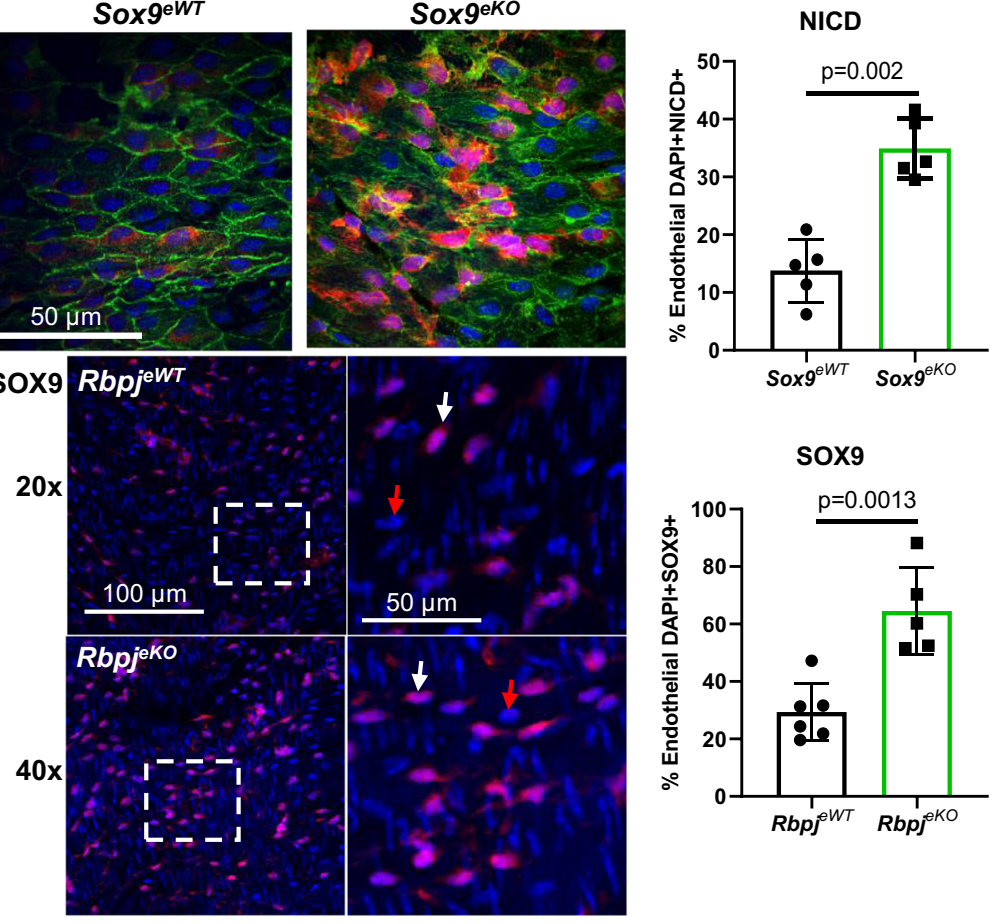

c
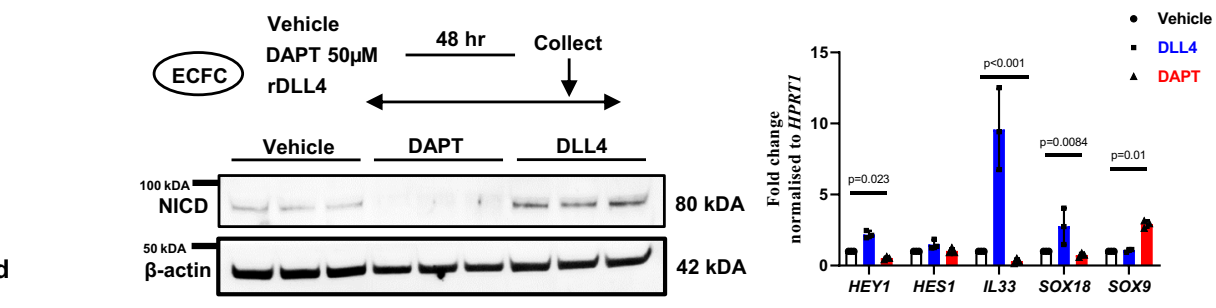

d

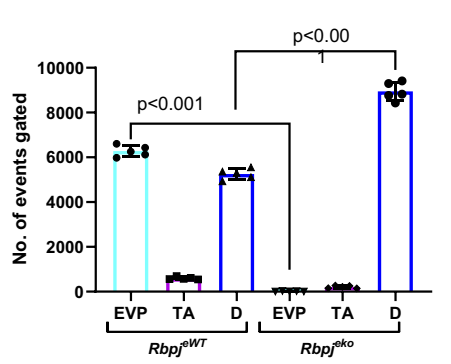

We therefore sought to identify if a reciprocal interplay could be observed between SOX9 and RBPJ that specifically mark the EVP population given the opposite phenotypes observed upon conditional deletion. Therefore, we used an endothelial specific knockout of $R b p j^{e K O}\left(R b p j^{f l f l} / C d h 5-C r e^{E R} R o s a Y F P\right)$. Here, following 10 days of tamoxifen injections, aortic tissue was harvested and evaluated under flow cytometry and qPCR (Fig. 5a).
qPCR demonstrated the abrogation of Rbpj expression as well as canonical Notch signalling, since Hes 1 and Hey 1 were markedly reduced in the entire endothelium $(* * * p<0.001$; Fig. 5a). Following $R b p j^{e K O}$, the expression of Sox 9 also increased by over 7-fold compared to controls. Moreover, $R b p j^{e K O}$ displayed a significant increase in EndMT genes Snail, Slug and Twist $\left({ }^{* *} p<\right.$ 0.01 ; $* * * p<0.001$; Fig. $5 \mathrm{~A})$. Genes involved in endothelial 
Fig. 5 Co-inhibitory interplay of SOX9 and Notch signalling in the endothelium. a Schematic diagram demonstrating experimental set up using conditional vascular specific Rbpj knockout mice (Rbpj fl/fl/Cdh5-Cre ${ }^{E R} R o s a Y F P-R b p j e K O$ ). qPCR analysis of key endothelial, quiescence and EndMT genes in

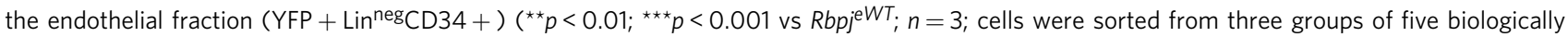
independent animals; mean $\pm \mathrm{SD}$; $p$ value was calculated by two-way ANOVA with multiple comparison of row mean). $\mathbf{b}$ Aortic whole-mount en face staining for activated-NOTCH1 intracellular domain (NICD) demonstrates significant increase in the nuclear localisation of activated-NICD in the endothelium after conditional deletion of SOX9. The deletion of RBPJ resulted in a significant increase in the number of SOX9 positive endothelial cells. ${ }^{* \star} p<0.01,{ }^{\star \star \star} p<0.001$ vs eWT; $n=4$ aorta from four biological independent animals; mean \pm SD; $p$ value was calculated by two-tailed unpaired $t$ test). c Western blot analysis of human endothelial colony forming cells, ECFCs shows DAPT inhibits activated-NICD accumulation, whereby rDLL4 treatment significantly upregulates activated-NICD expression showing the possibility to modulate canonical Notch signalling in vitro in ECFCs. qPCR analysis of key NOTCH pathway genes and SOX9 after modulation of canonical Notch pathway $\left({ }^{\star} p<0.05{ }^{\star \star} p<0.01 ;{ }^{\star \star \star} p<0.001\right.$ vs Vehicle; $n=3$; cells were isolated from three biologically independent donors; mean \pm SD; $p$ value was calculated by two-way ANOVA with multiple comparison of row mean) $\mathbf{d}$ Using flow cytometry three distinct populations were observed based on CD31 and VEGFR2 expression (from left to right endovascular progenitor EVP; transit amplifying TA; definitive differentiated D) showing the endothelial hierarchy and changes between wild-type controls $\left(R b p j{ }^{e W T}\right)$ and $R b p j{ }^{j K O}$ mice after 10 days tamoxifen. Number of EVP is significantly reduced in $R b p j{ }^{\mathrm{eKO}}(p<0.001$ vs RbpjeWT; $n=3$ biologically independent animals; mean \pm SD; $p$ value was calculated by two-way ANOVA with multiple comparison of row mean).

quiescence such as $I l 33, p 16, p 21$ and $p 57$ were also significantly downregulated $(* * * p<0.001)$. We further explored this opposite regulation of Sox9 and RBPJ/Notch signalling (Fig. 5b). In Sox $9^{e K O}$ aortas, there was a significant increase in nuclear NOTCH intracellular domain (NICD) reflecting the activation of canonical NOTCH signalling. Indeed over $30 \%$ of nuclei displayed nuclear NICD in Sox $9^{e K O}\left(p=0.002\right.$ vs Sox $9^{e W T}$; Fig. 5b). In contrast, $R b p j^{e K O}$ aortas displayed significantly higher levels of SOX9 expression as compared to controls $(p=0.0013$ vs $R b p j^{e W T}$; Fig. 5b). These findings corroborate at the protein level and in vivo, with the results obtained through qPCR to suggest that RBPJ negatively regulates SOX9 expression and that SOX9 negatively regulates canonical NOTCH signalling as well as RBPJ itself. We further tested this relationship in vitro. Human endothelial colony forming cells (ECFCs) were exposed to either vehicle control or to either the NOTCH ligand DLL4 coated plates or to the canonical NOTCH pathway gamma-secretase inhibitor DAPT (Fig. 5c). Each intervention had the expected outcome in terms of NOTCH pathway activation, NICD accumulation and affected gene expression of downstream effectors such as $H E Y 1$, or other genes important for endothelial function such as IL33 or SOX18. This system allowed us to explore the effect of canonical NOTCH signalling on SOX9 expression. DAPT treatment resulted in a significant increase in SOX9 ( $p=0.01$ vs Vehicle; Fig. $5 c$ ) supporting the claim that active NOTCH signalling and RBPJ negatively regulate Sox9 expression. However, DLL4 treatment and NOTCH pathway activation did not further affect SOX9 expression.

Similar to Sox $9^{e K O}$, a depletion in the EVP population in the aorta of $R b p j^{e K O}$ compared to $R b p j^{e W T}$ controls was observed under flow cytometry $(* * * p<0.001$; Fig. 5d). Similarly, in wounds at D5, conditional ablation of $R b p j e K O$ resulted in a reduction in EVPs. However, unlike Sox $9^{e K O}$ animals, in $R b p j^{e K O}$ the number of $\mathrm{D}$ cells in the granulation tissue was significantly reduced $(* * * p<0.001$; Supplementary Fig. 6b). Additionally, amongst Lin-YFP +, there was a significant increase in $M$ (CD34-CD31-) cells in $R b p j^{e K O}$ compared to controls, in line with the increased EndMT and fibrosis previously reported in these mice $\left(p=0.02 \text { vs } R b p j^{\text {eWT }} \text {; Supplementary Fig. } 6 \mathrm{c}\right)^{9}$.

These findings suggest that Sox 9 and Rbpj genetically interact via a negative feedback loop and drive opposite EndMT outcomes upon expression in the endothelium. Of significant interest, in the endothelial hierarchy described in both murine ${ }^{10}$ and human $\mathrm{ECs}^{9}, \mathrm{EVPs}$ in mice and meso-endothelial progenitors in human placenta have higher levels of expression of Sox 9 whereas fully differentiated D cells or ECFCs have high Notch signalling. This duality brought us to consider EVPs as a progenitor prompt to a fate choice towards either endothelium through increased Notch signalling (TA and D cells) or towards EndMT through increased Sox9 activity.

Activation of $\mathbf{H H}$ signalling drives EndMT. HH signalling has been implicated as a key regulator of Sox9 expression and function driven by GLI1 translocation into the nucleus. Additionally, previously published RNA sequencing data from our lab showed that Gli1 is significantly upregulated only in the EVP population of the murine endothelium ${ }^{10,13}$. In a different context, $\mathrm{HH}$ signalling has also been shown to reduce Notch signalling. To assess the molecular function of $\mathrm{HH}$ signalling in the endothelium we generated a Ptch1 endothelial specific knock-out that we termed a Ptch $1^{e K O}$ (Ptch $1^{f l / f l} / C d h 5-C r e^{E R}$ RosaYFP) to specifically drive HH signalling gain of function in the endothelium. Both Ptch $1^{e W T}$ and Ptch $1^{e K O}$ were provided tamoxifen injections and the aortic tissue was harvested for assessment of the endothelium (Supplementary Fig. 7a). To firstly assess if $\mathrm{HH}$ signalling was activated, YFP + EVP and D cells were FACS sorted from Ptch $1^{e K O}$ for $\mathrm{qPCR}$ analysis. Ptch1 was significantly reduced, whereas Smo, Gli1 and Sox9 were all significantly upregulated in the EVPs in comparison to $\mathrm{D}$ in the $P t c h 1^{e K O}$ ( $p<0.001$ vs D cells Ptch $1^{e K O}$; Supplementary Fig. 7b). The same was also observed when comparing EVP from Ptch $1^{e K O}$ to EVP from Ptch $1^{e W T}\left(p<0.001\right.$ EVP Ptch $1^{e W T}$ vs EVP Ptch $1^{e W T}$ Supplementary Fig. 7b). We then analysed the aortic endothelium under flow cytometry. The Ptch $1^{e K O}$ mice almost half (48\%) of all Lin-YFP + cells had lost CD34 expression, compared to only $13 \%$ for Ptch $1^{e W T}$ suggesting an accelerated loss of endothelial phenotype (Supplementary Fig. 7c). To understand differences in phenotypes between the different conditional knockout models, Ptch $1^{e K O}$, Sox $9^{e K O}$ and Rbpj1 $1^{e K O}$ aortas were compared to controls (Cdh5-Cre $e^{E R}$ RosaYFP). Despite slight differences, 4 weeks after tamoxifen injection, there was no significant changes in aortic endothelial numbers (as a proportion of live cells, Fig. 6b). All three conditional gene deletions resulted in strong reductions in EVP numbers, suggesting they affected progenitor function. However, they affected differently the other endothelial populations. The most dramatic changes occurred upon loss of rbpj resulting in increases in D and $\mathrm{M}$ cells. Loss of ptch 1 also resulted in increased $\mathrm{M}$ cells, whereas sox 9 loss resulted in a slight increase in $\mathrm{D}$ cells but did not significantly affect the endothelial cell phenotype distribution in homoeostasis. Overall, this showed that despite similar effects on EVPs, these different genes regulate endothelial cell fate slightly differentially during homoeostasis (Fig. 6C).

This also suggested that upon $\mathrm{HH}$ activation both $\mathrm{D}$ cells and $M$ cells could be obtained to explore the duality between Sox9 and Notch signalling. We therefore FACS sorted Lin-YFP + CD34+ (to reflect mostly D cells, given the major reduction in EVPs) and 
a

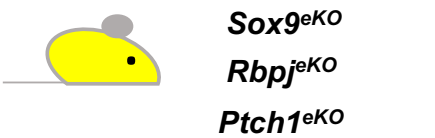

Tamoxifen

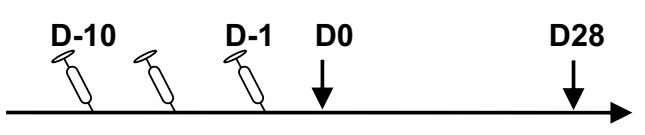

b
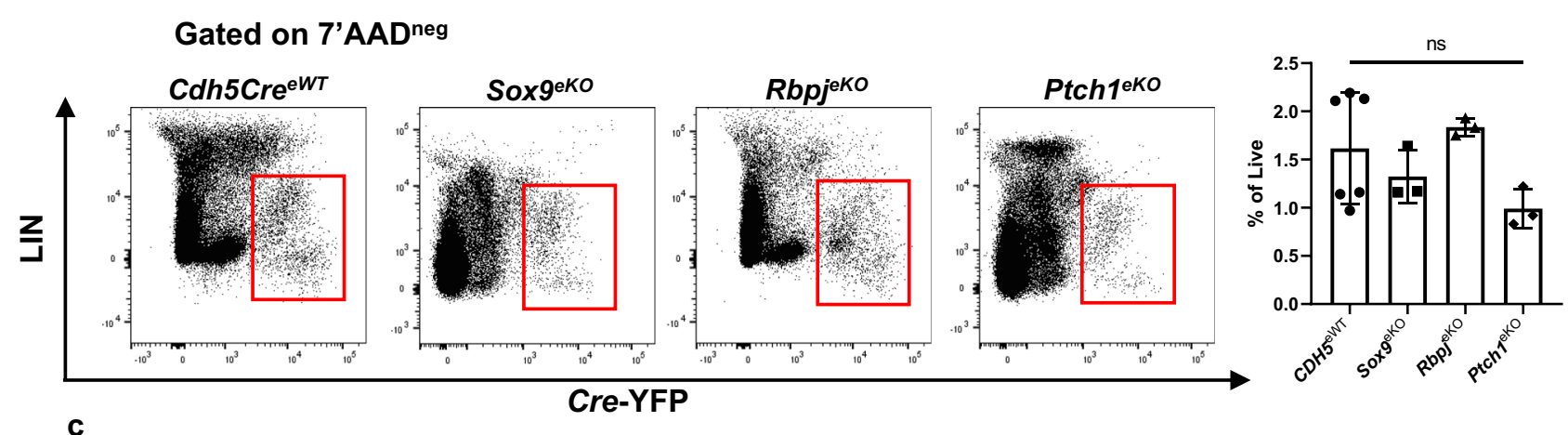

C
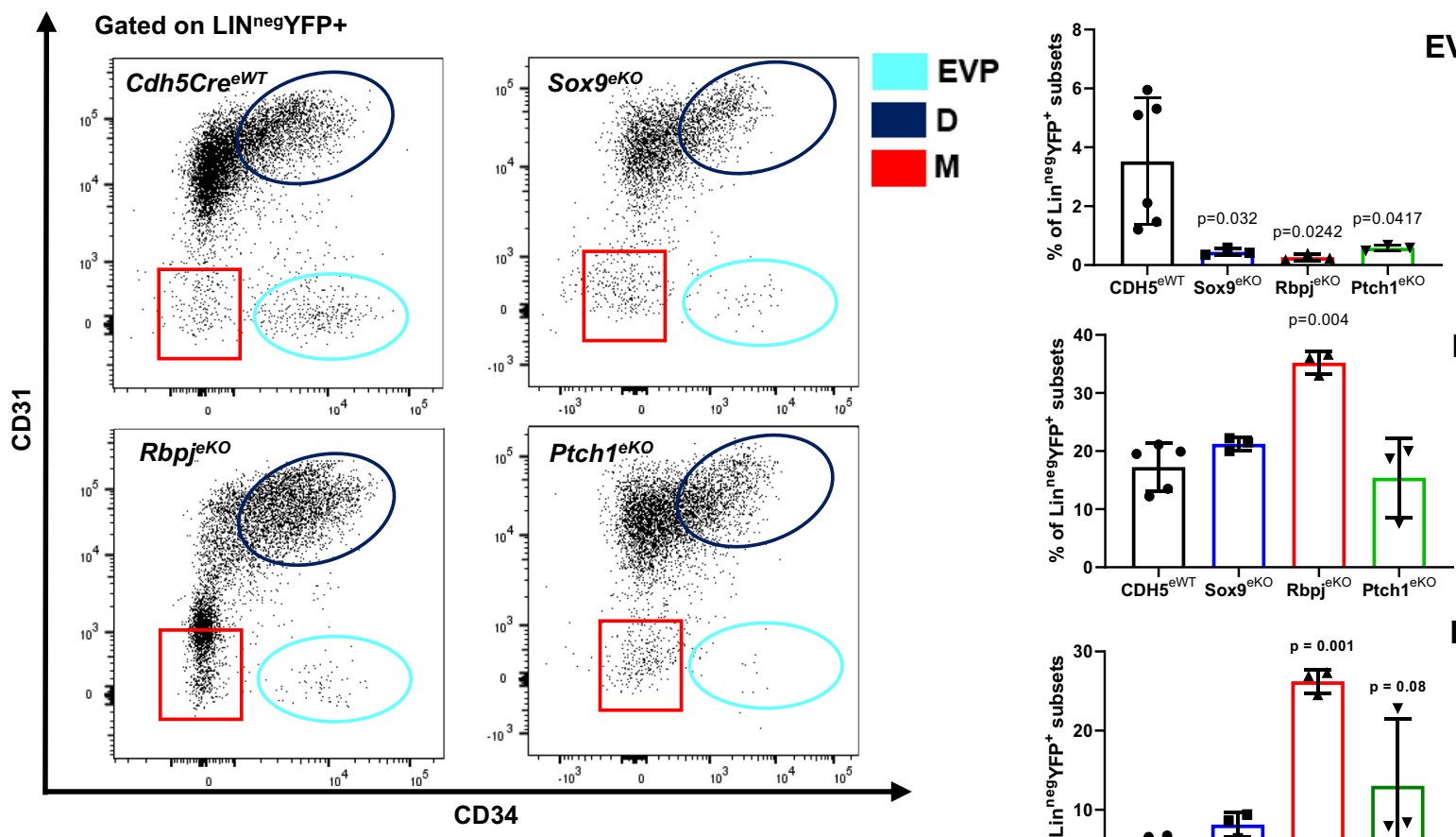

EVP
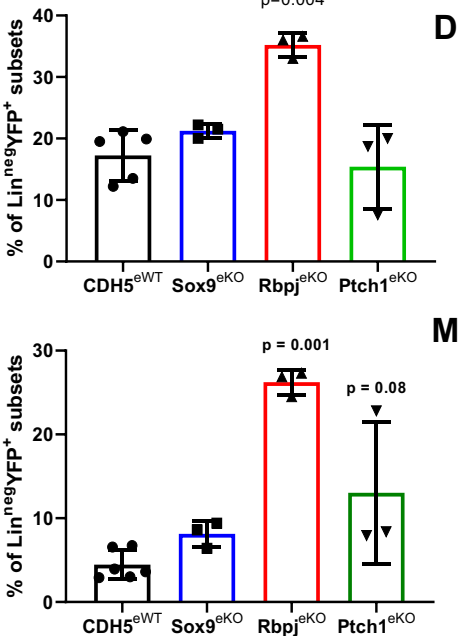

Fig. 6 Conditional deletion of key factors in the endothelium drives aortic progenitor directional fate-choice differentiation. a Schematic diagram demonstrating experimental set up of conditional Sox $9^{e K O}, R_{b j j}{ }^{\mathrm{eKO}}$ or Ptch ${ }^{e K O}$ knockout mice. b Flow cytometry plots showing the total endothelial population (YFP + Lin ${ }^{\text {neg }}$ ) within the aorta was not affected by the conditional deletion of Sox9, Rbpj or Ptch1 (ns $p>0.05$ vs eWT; $n=3$ biologically independent animals; mean $\pm \mathrm{SD}$; $p$ value was calculated by one-way ANOVA with multiple comparison to eWT). c Subsequent populations were then demarcated by CD31 and CD34 expression, demonstrating the significant depletion of EVPs in all the knockout mice compared to wildtype with different fates adopted by endothelial cells such as increases within the M population after the deletion of Rbpj and Ptch1 $\left({ }^{\star} p<0.05,{ }^{\star \star} p<0.01\right.$ vs eWT; $n=3$ biologically independent animals; mean $\pm \mathrm{SD} ; p$ value was calculated by one-way ANOVA with multiple comparison to eWT).

Lin-YFP + CD34- (to mostly reflect mesenchymal M cells) cells for analysis by qPCR. Notch signalling target genes (Rbpj, Hey1, Hes1) and endothelial gene Pecam1 were significantly increased in expression in the CD34 + 'endothelial' group and EndMT genes showed only slight reduction. However, in the CD34- 'mesenchymal' group, Notch family gene expression and Pecam was completely abrogated by over fourfold reduction in expression, however Sox9 expression was significantly increased along with the expression of downstream EndMT genes (Snail, Slug, Twist1, Twist $)\left(p<0.005\right.$ vs Ptch $1^{e W T}$; Supplementary Fig. $\left.7 \mathrm{~d}\right)$. These data further show the importance of the opposing role of SOX9 and Notch signalling in two different endothelial fates and highlight the role of $\mathrm{HH}$ signalling in this fate decision.

Increased $\mathrm{HH}$ signalling in the endothelium results in scarring and increased EndMT in skin wounds. To evaluate whether an increase in $\mathrm{HH}$ signalling results in accelerated EndMT and thus exacerbating fibrosis, as expected through the increase of SOX9 and decrease of Notch signalling, we again used the full thickness 

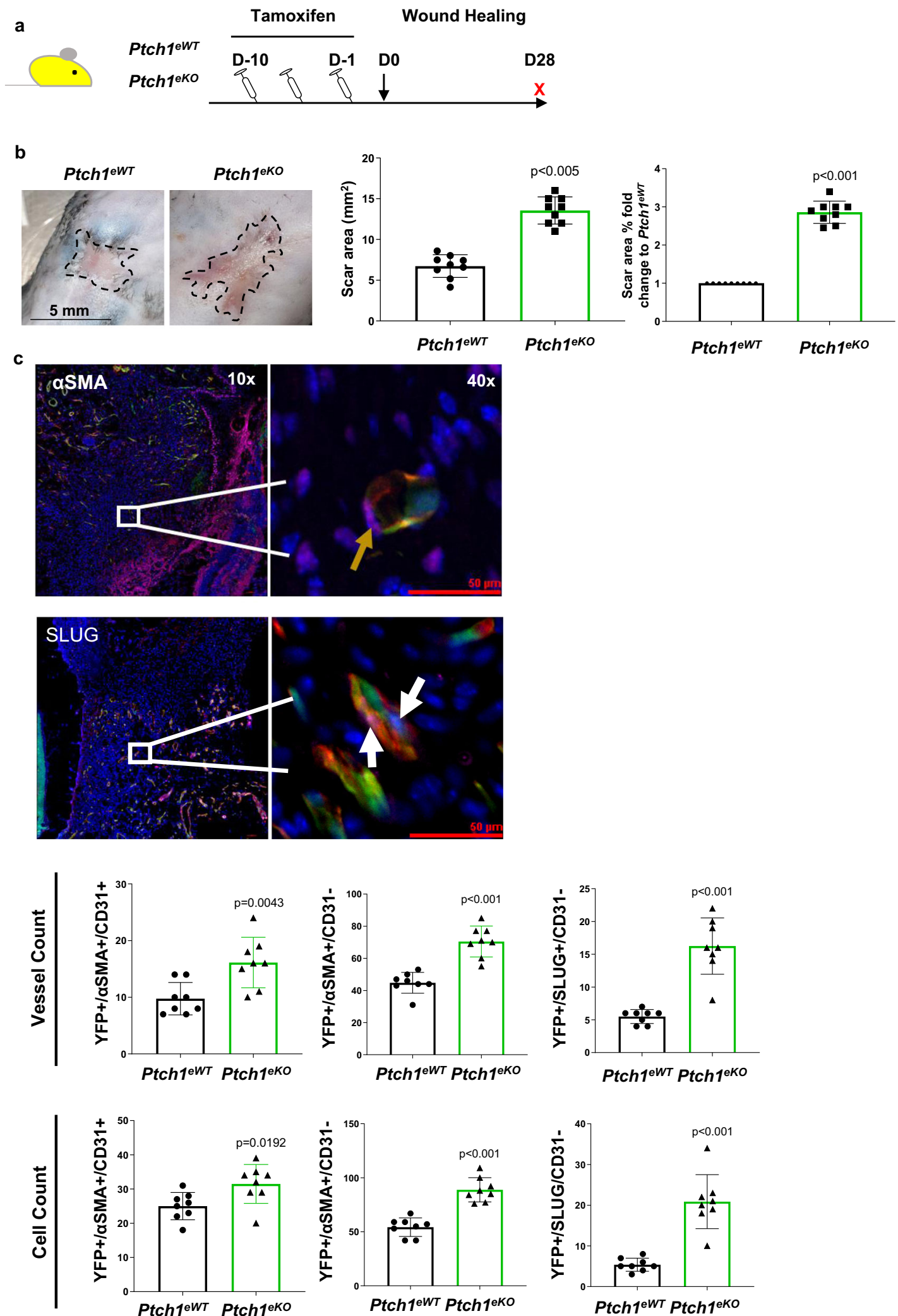

skin excisional $(1.5 \mathrm{~cm} \times 1.5 \mathrm{~cm})$ wounds on the dorsal flanks on the mice. Prior to wounding both Ptch $1^{e W T}$ and $P t c h 1^{e K O}$ were provided tamoxifen and wounds were collected at day 28 (D28) (Fig. 7a). At D28, macroscopic observation and quantification demonstrated the scar areas were over threefold larger in the Ptch $1^{e K O}$ compared to controls ( $p<0.005$ vs Ptch $1^{e W T}$; Fig. $7 \mathrm{~b}$ ). We then conducted $6 \mathrm{~mm}$ full thickness punch biopsy wounds on the dorsal flanks of the Ptch1 $1^{e W T}$ and Ptch ${ }^{e K O}$ mice following 10 days of IP tamoxifen injections. Wounds were then harvested at day 7 (D7) and assessed for EndMT using SLUG and $\alpha$-SMA. As expected, a significant increase in YFP + vessel and cell counts expressing both SLUG and a-SMA were observed in Ptch $1^{e K O}$ wounds compared to controls. Importantly, this also coincided with a significant increase in YFP $+\alpha \mathrm{SMA}+(p=0.0043$ vs 
Fig. 7 Increased Hedgehog signalling in the endothelium results in scarring and increased endothelial to mesenchymal transition (EndMT) in skin

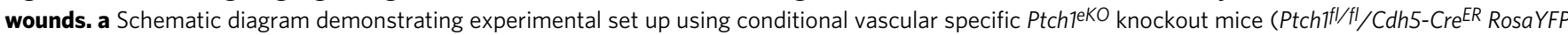
- Ptch ${ }^{K K O}$ ). b Photo images and scar surface assessment showing increase scar area at day 28 (D28) post wounding between wild-type controls

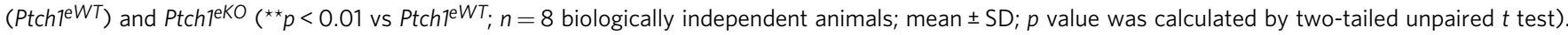
c Quantification of skin wound sections at day 7 (D7) with endothelial markers CD31 and YFP and EndMT markers SLUG and $\alpha$ SMA. High magnification representative images show YFP + (green) nuclei co-expressing $\alpha$ SMA (purple) and CD31 (red) marked by white arrow. Additionally, YFP + nuclei exhibit nuclear expression of SLUG (yellow arrow) $\left({ }^{\star \star} p<0.01 ;{ }^{* \star} p<0.001\right.$ vs Ptch $7{ }^{e W T} ; n=8$ biologically independent animals; mean \pm SD; $p$ value was calculated by two-tailed unpaired $t$ test).

Ptch $1^{e W T}$; Fig. 7c) and YFP + SLUG + vessels and single cells $\left(p<0.001\right.$ vs Ptch1 ${ }^{e W T}$; Fig. 7c) entirely losing CD31 expression.

Reduced scarring upon use of siRNA to inhibit Sox9 in wound healing. siRNA as a topical agent in treating wounds to improve healing and/or to reduce scarring have been used previously in clinical trials ${ }^{30}$. To evaluate the potential benefit of targeting Sox9 to reduce EndMT in wounds, we delivered siRNA against Sox9 using a topically applied pluronic gel directly onto full thickness skin excisional $(1.5 \mathrm{~cm} \times 1.5 \mathrm{~cm})$ wounds on the dorsal flanks of wild-type $(\mathrm{C} 57 \mathrm{Bl} / 6 \mathrm{~J})$ mice. The gel was applied twice daily between days 5-14 (D5-D14) and wounds harvested at D28 (Fig. 8a). The wounds and mice were closely monitored, with images taken every few days. Mice treated with Sox9 siRNA displayed enhanced wound healing and accelerated closure by D5, mirroring our observations in the Sox $9^{e K O}$ used earlier (Fig. 8b; $n$ $=8$ per group). Additionally, when scar area was assessed there was a $38 \%$ reduction following Sox 9 siRNA treatment compared to scrambled controls (Scr) $(p<0.001$ vs Scr; Fig. $8 c)$. EndMT was then quantified using $6 \mathrm{~mm}$ full thickness punch biopsy wounds on the dorsal flanks of mice. Mice were treated with topical pluronic gel twice daily from D1-6 with wounds harvested on D7. There was a significant reduction in CD31 + vessels and cells expressing either SLUG or a-SMA in mice treated with Sox9 siRNA ( $p<0.001$ vs Scr; Fig. $8 d$ ). Overall, the results of this study support the concept for blocking Sox 9 expression in the vasculature during wound healing to block EndMT and reduce scar area.

\section{Discussion}

The profound adaptive and regenerative capacity of the adult endothelium are attributed to a population of stem or progenitor cells that resides within the vasculature throughout all organ systems ${ }^{10,11,31,32}$. However, a functional and molecular definition of vascular endothelial progenitors remains in infancy. The description of Sox 9 and $R b p j$ as important TFs in demarcating EVP has potentiated more precise investigation into the mechanisms underlying vascular endothelial regeneration in homoeostatic turnover and pathological EndMT ${ }^{9,10}$. Specifically, this project employed functional fate-mapping in vivo and gene expression analyses to determine the effect of EVP fate choice during vascular homoeostasis and pathology. Here we show that modulation of Sox9 expression in the endothelium through its conditional knockout or its upregulation via reduced Notch signalling or increased $\mathrm{HH}$ signalling affects EVP fate choice between an endothelial or a mesenchymal phenotype. Increased expression of Sox 9 was associated with increased EndMT and fibrosis whereas Sox9 conditional deletion substantially reduced EndMT and scarring (Fig. 9).

A major finding in the present study was the molecular modulation of endothelial fate. As the key TFs modulating this fate are expressed at significantly higher levels in EVPs as compared to other endothelial populations, one can assume that mesenchymal transition as well as endothelial differentiation emanates from the progenitor EVP cells rather than D cells that do not express Sox9. We and others have described the existence of bipotent endothelial progenitors in the human term placenta able to give rise to both endothelial and mesenchymal colonies in vitro 9 . Of interest, the transcriptional profile as well as the surface expression of these cells is highly reminiscent of EVPs. Similarly, others have reported bipotent endothelial progenitors in mice ${ }^{33}$ or upon derivation from iPSCs ${ }^{34,35}$. Our study is limited by the inability to directly trace the fate of EVPs and D cells independently to demonstrate their potency. However, given the gene expression profile of Sox9, our findings suggest that EVPs have the capacity to evolve towards an endothelial phenotype and contribute to blood vessel formation or to enter mesenchymal transition towards myofibroblasts and contribute to scarring.

Trans-differentiation of endothelial to mesenchymal cells through EndMT has recently been associated with fibrotic disease in diverse cellular contexts, including the exacerbation of cardiac, pulmonary fibrosis and atherosclerotic plaque instability ${ }^{3,29}$ Using a variety of lineage tracing models and pro-fibrotic markers, ECs were identified to contribute to fibrosis by transitioning into pro-fibrotic mesenchymal cells, specifically fibroblasts and myofibroblasts $3,9,10$. Therefore, an understanding of the molecular basis of EndMT in diverse niches may clarify the clinical impact of EndMT and identify therapeutic targets. Although not previously associated with EndMT, Sox 9 has been attributed to the abnormal ECM remodelling in the kidney, cardiac and liver fibrosis $^{3,20,21}$. In kidney fibrosis, TGF- $\beta$, a known major regulator of the EndMT pathway, was shown to upregulate Sox9 expression exacerbating tissue fibrosis and leading to further kidney disease ${ }^{3}$ whereas here we show that Sox9 deletion also affected TGF $\beta$ downstream targets. Sox 9 is also vital for mesenchymal stem cell maintenance, acting as a key regulator in differentiation into adipocyte, chondrocyte and osteocyte lineages ${ }^{36,37}$. As Sox9 is described to regulate both stem cell maintenance and mesenchymal differentiation, its upregulation within EndMT suggests a dual role for Sox9 to maintain both EVP stemness and a mesenchymal fate plasticity. Our study demonstrated the importance for Sox9 in maintaining EVP in a quiescent state as well as maintaining colony-formation capacity. Its loss resulted in rapid transition from a progenitor to $\mathrm{D}$ fate but more importantly a significant reduction in scar tissue in the skin wound healing scenario. Although, the conditional loss of RBPJ produced a similar reduction of EVP and self-renewal capacity, there was a significantly different and often opposing fate resulting from EVP differentiation towards mesenchymal cells. This was also reproduced upon conditional Ptch1 deletion. Overall, these findings highlight the importance of Sox9, Notch and $\mathrm{HH}$ pathways in EVP function. However, loss of these pathways results in unique changes in cell fate.

The opposing phenotypes observed upon conditional loss of Sox 9 or Rbpj supported a negative regulatory role between these two pathways. Indeed, in wounds, Sox9 deletion resulted in decreased $M$ cells emanating from EndMT whereas Rbpj deletion increased mesenchymal transition and scarring. At the molecular level both in vitro and in vivo, negative regulation of canonical 
a

Wound Healing

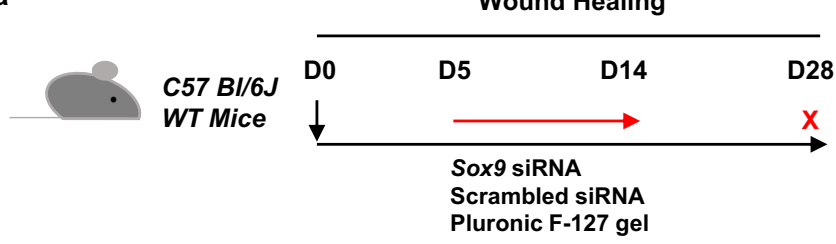

b
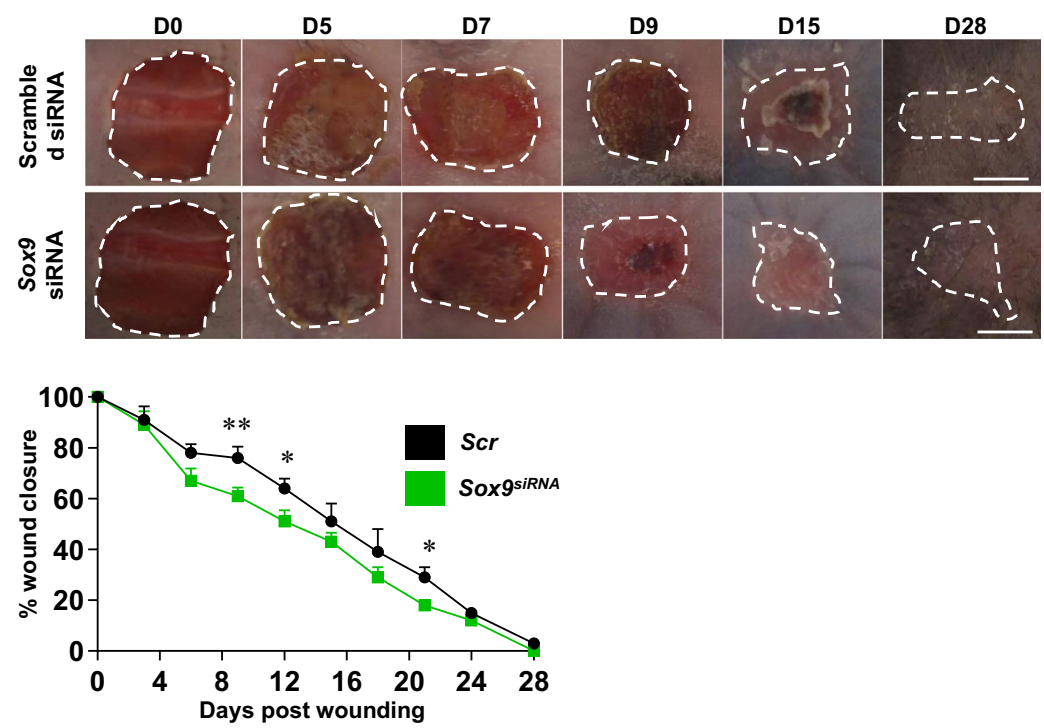

C
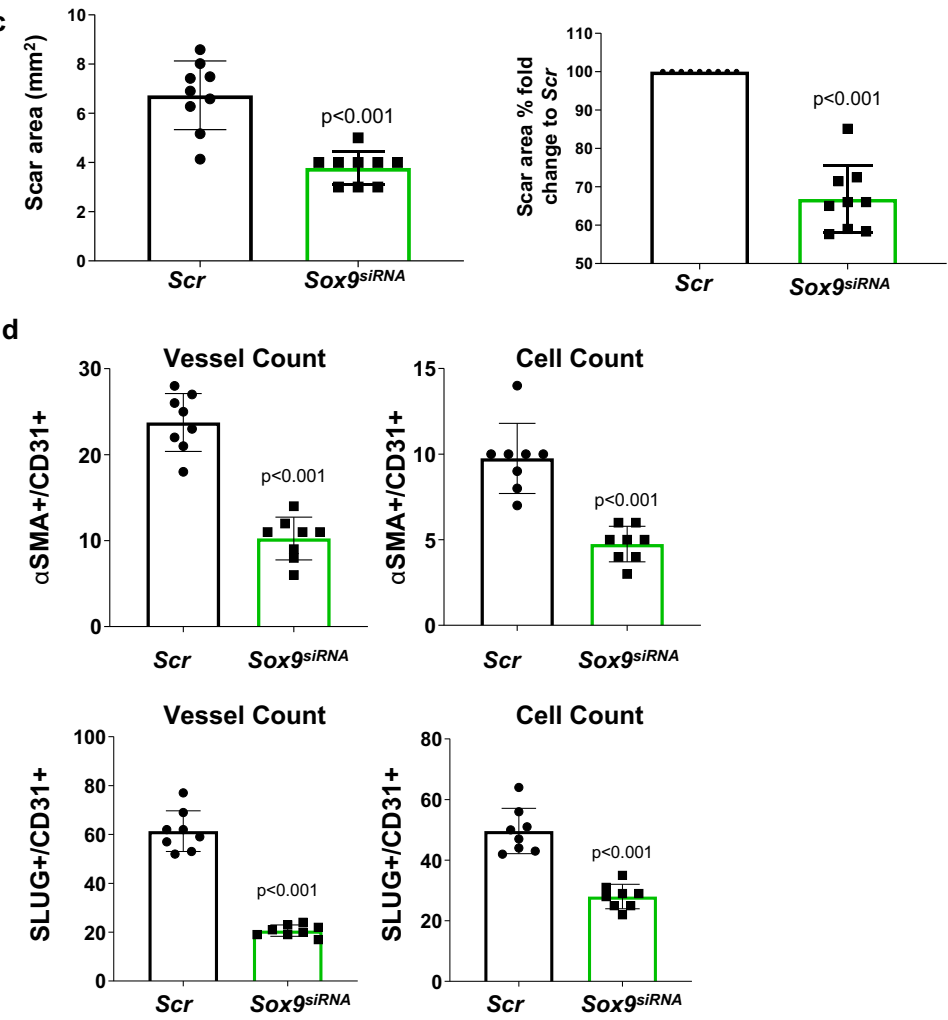

Fig. 8 Reduced scarring upon use of siRNA to inhibit Sox9 in wound healing. a Schematic diagram demonstrating experimental set up using wild-type mice. b Photo images showing wound healing over the course of 28 days (D28) post wounding between scrambled (Scr) controls and Sox 9 siRNA (Sox9siRNA) treated mice. Significant increase in wound healing was observed in mice treated with (Sox9siRNA) compared to Scr ( ${ }^{\star} p<0.05 ;{ }^{\star \star} p<0.01$ vs $\operatorname{Scr}$; $n=8$ biologically independent animals; mean \pm SD; $p$ value was calculated by two-way ANOVA with multiple comparison of row mean). c Significant

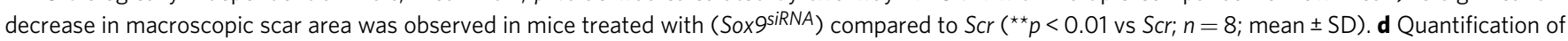
skin wound sections at day 7 (D7) with endothelial markers CD31 and EndMT markers SLUG and $\alpha$ SMA (** $p<0.01 ;{ }^{\star \star \star} p<0.001$ vs Scr; $n=5$ biologically independent animals; mean $\pm \mathrm{SD} ; p$ value was calculated by two-tailed unpaired $t$ test). 


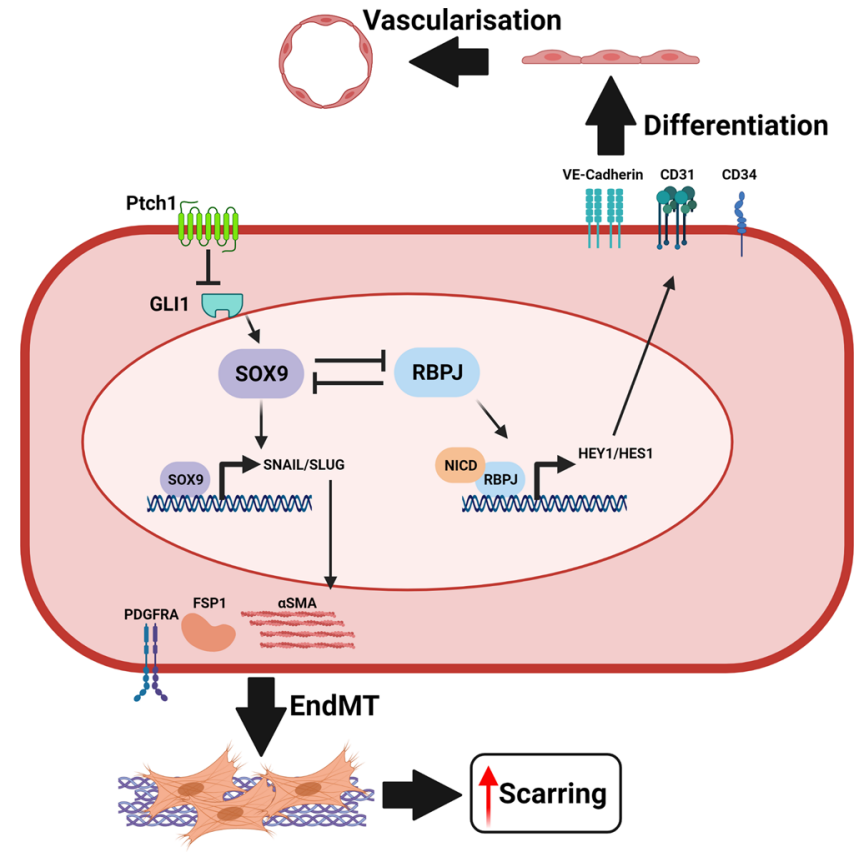

Fig. 9 Regulation of endovascular progenitors cell fate. The fate of EVPs is maintained by the co-inhibitory relationship between SOX9 and RBPJ maintained in a progenitor state in homoeostasis. However, increased expression of SOX9, either through the loss of RBPJ, or activation of the hedgehog pathway results in the translation of SNAIL and SLUG, driving the expression of mesenchymal-related factors such as FSP1, $\alpha$ SMA and PDGFRA. Thus, triggering EndMT and excessive scar formation.

Conversely, the deletion of SOX9 promotes Notch signalling and RBPJ activities, favouring endothelial differentiation and vascularisation.

Notch signalling increased Sox9 expression at RNA and protein level. Similarly, Sox9 deletion in vivo resulted in increases in canonical Notch signalling. However, further increase in this signalling did not result in Sox9 reduction, possibly suggesting that Sox9 gene negative regulation is exerted by RBPJ alone. This duality could be further visualised in the Ptchl conditional ablation model. Specifically, the mesenchymal population upregulated Sox9 and fibrosis-associated TFs and downregulated Notch signalling as well as genes associated with endothelial fate. In contrast, in the same mice, ECs had high levels of Notch signalling and had downregulated Sox9. It remains unknown whether the HH effectors, Gli1 and Gli2, directly or indirectly regulated target genes.

The opposing fates adopted by ECs in the context of wound healing could be defined by markers of Notch signalling, Heyl and Hesl, which have been extensively described in human and murine endothelial biology. Specifically, Notch signalling integrates with cyclin-dependent kinase inhibitors p16, p21 and p57 for quiescent progenitor maintenance, and endothelial attachment genes including CD31 and VE-Cadherin to maintain endothelial function ${ }^{22,25}$. Notably, the downregulation of Notch signalling in this study revealed a loss of endothelial self-renewal characteristics and endothelial quiescence genes such as $I l 33^{25}$. In contrast, upregulation of EndMT-associated genes in the transitioning mesenchymal population further elucidates the contribution of $\mathrm{HH}$-activation to EndMT by indicating transcriptional biomarkers for a more complex mesenchymal transition. For example, Snail has predominantly been associated with pathological fibrosis downstream of the classical TGF $\beta$ pathway, whereby the recruitment of Twist transcriptional regulators drive the further upregulation of motility genes such as $\alpha S M A$ and matrix metalloproteases, and excess deposition of fibrotic collagen subtypes $^{34,38-40}$. Furthermore, Snail stabilised by GSK-3 $\beta$ is demonstrated to induce osteogenic EndMT contributing to atherosclerotic calcification in response to the cardiovascular disease risk factor, oxLDL ${ }^{3,41}$. Our group has previously identified the notion of a resident endothelial progenitor cell having probable biopotential capacity9. Shafiee et al., identified a novel mesoendothelial cell from term placental tissue that demonstrated Notch signalling to be crucial in maintaining endothelial phenotype. In contrast however, differentiation to a mesenchymal phenotype was not via the classical TGF $\beta$ EndMT pathway. This has also been previously shown in situations of EndMT, whereby Slug is activated independently from TGF $\beta$ and Snail directly by Notch signalling pathway in the initiation of cardiac cushion EndMT ${ }^{42-44}$. Slug function is further dependent on cooperative Sox 9 protein-binding to induce TGF $\beta$-mediated EndMT progression, consistent with the upregulation of Sox9 in EndMT as identified here ${ }^{45}$. Previous literature has reported an interdependent protein interaction between SLUG and SOX9 in mammary stem cells, human lung carcinoma and kidney fibrosis ${ }^{27,45,46}$, whereby inhibition of either SLUG or SOX9 resulted in inhibition of the mammary bipotent stem cells or cancer cells ${ }^{27,45}$. This highlights the complexity of bipotential differentiation, therefore elucidating differentiation pathways will aid in better understanding tissue regeneration and/or disease initiation.

Currently, there are over 20 clinical trials utilising siRNA to silence aberrant genes in a variety of pathologies including cancer, atherosclerosis and hypertrophic scarring ${ }^{47}$. Similarly, Sox9 siRNA has been previously shown to be efficient in the knock-down of Sox9 in cancer and other pathologies ${ }^{48,49}$. To administer the siRNA, Pluronic F-127 gel was utilised, which has proven effective in wound healing and drug delivery ${ }^{50,51}$. A significant reduction was detected in fibrotic scar area following Sox9 siRNA application in comparison to a scrambled siRNA administered control. Sox9 plays a vital role in the skin wound healing process due to its regulation of HFSCs (hair follicle stem cells), re-epithelisation of the wound tissue and stimulating ECM secretion via regulation of fibroblasts and myofibroblast populations ${ }^{34,47,52,53}$. In this study, we explored the possibility of a preventive therapy to avoid excessive scarring. It is unclear if Sox9 siRNA and inhibition of EndMT will have any impact on already established scars.

In conclusion, Sox9 expression in EVP is essential in maintaining quiescence and clonogenicity, however is also essential in driving pathological EndMT when a loss of Rbpj and Notch signalling is observed. Our findings strongly suggest that blocking Sox9 within EVPs is a valid strategy in reducing EndMT and vascular fibrotic disease.

\section{Methods}

Animals. All mice were maintained in accordance with University of Queensland ethics approvals and guidelines for care of experimental animals. Mice, regardless of sex (ages 10-12 weeks of age; genders housed separately) were used for this study. C57BL/6 mice (WT) were obtained from the Animal Resources Centre (Perth, Western Australia). All mice were group-housed (maximum of five mice per cage) and maintained under a regular light-dark cycle altered every $12 \mathrm{~h}$ with free access to water and standard mouse chow. The Cdh5-Cre ${ }^{E R t 2}$ mouse line was crossed with $\mathrm{ROSA}_{\text {lox }} \mathrm{YFP}_{\text {lox }}$ for endothelial-specific lineage tracing experiments. The subsequent double transgenic offsprings were termed Cdh5-Cre ${ }^{E R}$ RosaYFP. To induce endothelial specific knockout of the gene Sox9, Rbpj and Ptch1, Sox $9 f-f l$, $R b p j^{f l-f l}$ and $P t c h f^{f l-f l}$ mice then bred with Cdh5-Cre ER RosaYFP to create the tripletransgenic offsprings Sox $9^{f l / f l} / C d h 5-C r e^{E R}$ RosaYFP, Rbpj $f^{l / f l} / C d h 5-C r e^{E R}$ RosaYFP and $P t c h h^{f l f l} / C d h 5-C r e^{E R}$ RosaYFP, respectively. In all lineage tracing experiments, mice were subjected to a regimen of tamoxifen injection up to 10 days. Tamoxifen (Sigma-Aldrich, MI, USA) was dissolved in 90\% corn oil (Sigma-Aldrich) and $10 \%$ ethanol, with each mouse receiving a $2 \mathrm{mg}$ dose daily in $100 \mu \mathrm{L}$.

In vivo wound healing assay. Full thickness excisional wounds were created using a $6 \mathrm{~mm}$ sterile punch biopsy (Stiefel Laboratories, Research Triangle Park, NC) along the dorsal skin. Wounds were then left open with animals culled at each 
respective timepoints after wounding. In lineage tracing experiments, animals were injected with tamoxifen prior to wounding. Wound closure and total surface were manually defined, with the surface area recorded at individual timepoints. Measurements for the surface area were calculated using ImageJ software (National Institute of Health). To assess fibrosis and scar formation, large full thickness excisional wounds $(1.5 \times 1.5 \mathrm{~cm})$ were created. The wounds were left to heal for 28 days before the scar tissues were harvested and measured for scar area using ImageJ.

Skin wound harvest and processing. Mice were culled using $\mathrm{CO}_{2}$ asphyxiation. Organs and $6 \mathrm{~mm}$ wounds with $2 \mathrm{~mm}$ of surrounding skin were dissected and fixed in $4 \%$ paraformaldehyde (Sigma-Alrich, MO, USA) for $2 \mathrm{~h}$ at $4{ }^{\circ} \mathrm{C}$. Fixed samples were cyro-protected with incubations in $10 \%$ and subsequently $30 \%$ sucrose (Chem-Supply, SA, AUS) in phosphate buffered solution (PBS) overnight. The samples were embedded in Tissue-Tek Optimal Cutting Temperature (Sakura Finetek, CA, USA) in preparation for cyro-sectioning. Before embedding, wounds were cut in half to ensure that sections were taken from the centre of the granulation tissue, whereby $10 \mu \mathrm{M}$ sections were taken at $30 \mu \mathrm{M}$ intervals onto Superfrost Plus slides (Thermo Fisher Scientific, Waltham, USA) using a CM1905 Cyrostat (Leica Biosystems, Wetler, Germany). Slides were stored at $-30^{\circ} \mathrm{C}$.

Measurement of large wound scar surface area. Tile-scan images of scars were conducted using an Olympus BX51 Fluorescence microscope (Olympus, Tokyo, Japan) (bright-field) at $4 \mathrm{X}$ magnification, with the edge of the hairline taken as the edge of the scar. The scar surface area was measured in ImageJ and a fold change was calculated using the control.

PicoSirius Red staining and analysis. Wounds were stained using PicoSirius red staining (Invitrogen, CA, USA) as previously published ${ }^{9}$. Tile-scans of scars were imaged using an Olympus Upright Epi (Olympus, Tokyo, Japan) (bright-field) at 10X magnification. Images were analysed in Visiopharm (Visiopharm, Hørsholm, Denmark) software, where scar area was calculated using the in-built 'tune' tool, allowing the software to be taught to recognise the scar area, total scar area was then calculated.

Immunofluorescence. Tissue collected were fixed for $2 \mathrm{~h}$ at room temperature in $4 \%$ PFA. This was followed with $3 \mathrm{x}$ wash of $1 \mathrm{x}$ PBS (Amresco, Solon, Ohio, USA). Tissues were then infused under a sucrose gradient for cryo-protection before cryoembedding in Tissue-Tek ${ }^{\oplus}$ O.C.T. Compound (Sakura Finetek, Torrance, California, USA). Prior to antigen staining, cryo-sections were permeabilized in $0.5 \%$ TritonX-100 (Chem Supply, Gillman, South Australia) before blocking with 20\% normal goat serum. In this study, primary antibodies used includes rat anti-mouse CD31 (1:100), rabbit anti-aSMA (1:200), rabbit anti-FSP1 (1:200), rabbit antiSLUG (1:200), rabbit anti-SOX9 (1:500), rabbit anti-ERG (1:500) and mouse antiSOX9 (1:200). Primary antigen staining was conducted overnight at $4{ }^{\circ} \mathrm{C}$. Sections were then subjected to $3 \times 5 \mathrm{~min}$ washes in $1 \mathrm{x}$ PBS $+0.1 \%$ Tween-20 (Amresco, Solon, Ohio, USA). Secondary antibodies conjugated with Alexa-fluor 568 or 647 (Invitrogen, Carlsbad, CA, USA) were used for fluorescence detection. Sections were incubated with secondary antibodies for $40 \mathrm{~min}$ at room temperature. Nuclear staining was revealed in specimens mounted with ProLong ${ }^{\circledR}$ Gold mounting media containing DAPI (Invitrogen, Carlsbad, CA, USA).

En face aorta preparation. For en face aorta staining, mice were perfused with icecold $1 x$ PBS, then $4 \%$ PFA and a final perfusion of PBS. After, aortas were removed for further dissection and cleaning. Under a dissection microscope, attached diaphragm, vascular branches and perivascular adipose tissue were removed from the aorta. The aorta was then opened via longitudinal dissection by spring scissors and flatten with minutien pins onto dissection wax (Fine Science Tools, United States). Flatten aortas were fixed for $2 \mathrm{~h}$ at room temperature in $4 \%$ PFA. The fixative was removed with $3 \mathrm{x}$ washes of $1 \mathrm{x} \mathrm{PB}$, followed by blocking with $20 \%$ normal goat serum in $1 \mathrm{x}$ PBS. For en face staining, primary antibodies used included rat antimouse CD31 (1:100), rabbit anti-SOX9 (1:200) and rabbit anti-activated NICD (1:200). Subsequent secondary staining and mounting was carried out as described in the previous immunofluorescence section.

Microscopy. Confocal images were acquired with either the Olympus FV3000 confocal microscope or CSU-W1 SoRa Nikon Spinning Disk microscopes using Flouview FV3000 software v1.0 and NIS imaging software v4.5, respectively. Confocal images were acquired with a microscope equipped with Argon 561-10 nm DPSS and $633 \mathrm{~nm}$ HeNe lasers, and a 405-30 nm diode. Images were obtained at 20x, 40x and 100x. Immunofluorescence vessel quantification was conducted using ImageJ v1.53 and NIS elements v4.60.00. PSR collagen quantification was conducted using Olyvia v3.2.1.

Administration of siRNA. Sox9 and scrambled siRNA (control) (Dharmacon, VIC, AUS) were diluted to $3 \mu \mathrm{M}$ in Ultrapure distilled water (Table S1 for siRNA sequence). A 30\% w/v solution of Pluronic ${ }^{\circledast}$ F-127 (Sigma-Aldrich, MO, USA) and siRNA was made and administered twice daily directly onto large and small wounds using a pipette. In total, $75 \mu \mathrm{l}$ was administered to each large wound and all four small wounds. For large wounding experiments, mice started the siRNA administration from D5 to D12. For small wounding experiments, mice started the siRNA administration from D1 to D7.

Tissue processing and digestion. Tissues were collected for FACS analysis at defined specific end timepoints (D5 and 7). Aorta and skin wounds were first finely minced with scissors and then digested for $20 \mathrm{~min}$ at $37^{\circ} \mathrm{C}$ in an enzymatic cocktail containing $1 \mathrm{mg} / \mathrm{ml}$ collagenase I (Gibco, Life Technologies, NY, USA), $1 \mathrm{mg} / \mathrm{ml}$ dispase (Gibco, Life Technologies, NY, USA), $150 \mu \mathrm{g} / \mathrm{ml}$ DNase-I (Sigma-Aldrich, St Louis, MO, USA) in Hanks' Balanced Salt Solution. After digestion, the cell suspension was then passed through a $70 \mu \mathrm{M}$ cell strainer. Single cell suspensions were then used for FACS sorting or analysis by flow cytometry.

Flow Cytometry and FACS. Dissociated single cells in FACS buffer ( $0.5 \%$ BSA, 1 $\mathrm{mM}$ EDTA in $1 \mathrm{x}$ PBS) were then incubated with various antibody combinations for FACS analysis and sorting. A Gallios ${ }^{\mathrm{Tm}}$ flow cytometer was used for sample acquisition, with subsequent data analyses performed with Kaluza ${ }^{\circledast}$ analysis software (Beckman-Coulter, Miami, Florida, USA). A FACSaria cell sorter was utilised for FACS sorting using the FAVSDiva v5.0.3 software with subsequent analysis performed with FlowJo v10 (Becton Dickinson, Franklin Lakes, NJ, USA). Standard doublets discrimination and $7^{\prime} \mathrm{AAD}$ or FVS live/dead staining was carried out to only include live singlet events. The following combinations of antibodies were used to assess the endothelial hierarchy populations: Rat anti-mouse VEGFR2 PE (1:100), CD31 PE-Cy7 (1:1000) and CD34 Alexa647 (1:100) (Becton Dickinson, NJ, USA), Rat anti-mouse Lineage cocktail BV450 (1:50) (Biolgend), CD26 PE (1:200), CD140a BV605 (1:100) and Rat anti-mouse CD144 FITC (1:50) (eBioscience).

Cytospin of FACS sorted cells. Cells are FACS sorted directly into $2 \%$ FCS, and then washed with and resuspended in 1X PBS.A Cytospin ${ }^{\text {TM }} 4$ Cytocentrifuge was utilised for the distribution of cells in suspension onto SuperFrost Plus ${ }^{\mathrm{Tx}}$ slides. In short, cells were loaded into supplied columns and inserted into the centrifuge as per previously published protocol ${ }^{54}$. Adhered cells were then fixed with ice-cold $4 \%$ PFA and then blocked with $20 \%$ NGS. Standard immunofluorescent staining protocol described in the previous section was then carried out.

RNA Extraction, cDNA synthesis and qPCR. RNA was extracted from FACS sorted or cultured cells using a QIAGEN mini kit (Qiagen, Valencia, CA) according to the manufacturer's instructions. RNA quality and concentration was assessed using A260nm/A280nm spectroscopy on the Nanodrop ND-1000 (Thermo-scientific, Langenselbold, Germany). About 5-100 ng of RNA was used for cDNA synthesis using the Superscript III Reverse Transcription Kit (Invitrogen, Mount Waverley, Australia). For cDNA synthesis, collected RNA was treated with DNaseI (Sigma-Aldrich) to remove genomics DNA contamination. After which, cDNA synthesis was conducted using the Invitrogen SuperScript III First Strand Kit (Life Technologies, United States). cDNA was then treated with RNase H (Life Technologies). To quantify relative changes in key gene expression after treatment, real time-qPCR was conducted on cDNA using SYBR Green Master Mix reagent (Applied Biosystems, United Kingdom). Fold change of gene expression was determined using the delta delta Ct method, normalised to housekeeper gene Rpl5 in mouse or HPRT1 in human. Primer sequences are listed in Supplementary Table 2.

ECFC culture. Human term placentas were obtained after informed written consent from healthy pregnant women and placental foetal ECFC and MSC isolated using our previously published protocol ${ }^{24}$. The use of human tissue was granted by the human ethics boards of The University of Queensland and the Royal Brisbane and Women's Hospital. ECFCs were cultured on rat tail collagen coated tissue culture flasks in Endothelial Growth Medium (EGM-2, Lonza Group, Basel, Switzerland) with $10 \%$ of foetal bovine serum (FBS). MSCs were cultured using standard tissue culture flasks in DMEM with 10\% (FBS).

Western blots. Protein were obtained from cells lysed with radioimmunoprecipitation (RIPA) buffer supplemented with $1 \mathrm{x}$ complete protease inhibitor (Sigma-Aldrich) followed by sonication and centrifugation. SDS-PAGE of lysates was performed with the Bolt ${ }^{\mathrm{m}} 4$ to $12 \%$, Bis-Tris system (Invitrogen) and protein transfer was conducted with the Biorad Trans-Blot Turbo Transfer System according to the manufacturer's protocols. Immunoblots were labelled with following primary antibodies after blocking with Licor Intercept blocking buffer: rabbit anti-SOX9 (1:1000, Merck Millipore, Burlington, Massachuesetts, USA), rabbit anti-activated NOTCH1 (1:1000, Abcam) and mouse anti- $\beta$-actin (1:5000, Sigma-Aldrich). Primary antibodies were detected using fluorescently conjugated secondary antibodies: goat anti-rabbit IgG IRDye $800 \mathrm{CW}$ and goat anti-mouse IgG IRDye 680RN (1:2500, LI-COR Biosciences, Lincoln, Nebraska, USA). Detection and quantification of fluorescence intensity were performed using an Odyssey CLx imaging system (LI-COR Biosciences, Lincoln) and Odyssey 2.1 software or ChemiDoc MP Imaging System and ImageLab software v6.1. For an example of presentation of full scan blots, see Supplementary information. 
Statistical analysis and reproducibility. All statistical analyses were performed using GraphPad Prism v8 software. Data were analyzed using the following tests: two-tailed unpaired $t$-tests one-way or two-way ANOVA with Bonferroni correction. A $p$ value $<0.05$ was considered significant. For immunofluorescent and macroscopic quantification of granulation tissue, at least five slides were evaluated per wound, collected from a minimum of five biologically independent animals. Quantification of protein expression in the aortic endothelium was carried out with a minimum of five aortas from biologically independent animals. Quantification of protein expression from cytospun cells were carried out on cells sorted from a minimum of three groups of three biologically independent animals. Data were averaged per biological replicate for statistical analysis. For all quantification, single blinding was conducted.

Reporting summary. Further information on research design is available in the Nature Research Reporting Summary linked to this article.

\section{Data availability}

The data that support the findings of this study are available from the corresponding author upon reasonable request.

Received: 2 April 2020; Accepted: 23 March 2021; Published online: 07 May 2021

\section{References}

1. Aird, W. C. Endothelial cell heterogeneity. Cold Spring Harb. Perspect. Med. 2, a006429 (2012)

2. Okada, Y. et al. A three-kilobase fragment of the human Robo4 promoter directs cell type-specific expression in endothelium. Circ. Res. 100, 1712-1722 (2007).

3. Li, Y., Lui, K. O. \& Zhou, B. Reassessing endothelial-to-mesenchymal transition in cardiovascular diseases. Nat. Rev. Cardiol. 15, 445-456 (2018).

4. Kovacic, J. C. et al. Endothelial to mesenchymal transition in cardiovascular disease: JACC state-of-the-art review. J. Am. Coll. Cardiol. 73, 190-209 (2019).

5. Eisenberg, L. M. \& Markwald, R. R. Molecular regulation of atrioventricular valvuloseptal morphogenesis. Circ. Res. 77, 1-6 (1995).

6. de la Pompa, J. L. Notch signaling in cardiac development and disease. Pediatr. Cardiol. 30, 643-650 (2009).

7. Moonen, J. R. et al. Endothelial-to-mesenchymal transition contributes to fibro-proliferative vascular disease and is modulated by fluid shear stress. Cardiovasc. Res. 108, 377-386 (2015).

8. Zeisberg, E. M. et al. Endothelial-to-mesenchymal transition contributes to cardiac fibrosis. Nat. Med. 13, 952-961 (2007).

9. Shafiee, A., Patel, J., Hutmacher, D. W., Fisk, N. M. \& Khosrotehrani, K. Meso-endothelial bipotent progenitors from human placenta display distinct molecular and cellular identity. Stem Cell Rep. 10, 890-904 (2018).

10. Patel, J., Baz, B., Wong, H. Y., Lee, J. S. \& Khosrotehrani, K. Accelerated endothelial to mesenchymal transition increased fibrosis via deleting notch signaling in wound vasculature. J. Invest. Dermatol. 138, 1166-1175 (2018).

11. Wakabayashi, T. et al. CD157 Marks Tissue-Resident Endothelial Stem Cells with Homeostatic and Regenerative Properties. Cell Stem Cell 22, 384-397 (2018). e386.

12. Yu, Q. C., Song, W., Wang, D. \& Zeng, Y. A. Identification of blood vascular endothelial stem cells by the expression of protein C receptor. Cell Res. 26, 1079-1098 (2016).

13. Lukowski, S. W. et al. Single-cell transcriptional profiling of aortic endothelium identifies a hierarchy from endovascular progenitors to differentiated cells. Cell Rep. 27, 2748-2758.e2743 (2019).

14. Donovan, P. et al. Endovascular progenitors infiltrate melanomas and differentiate towards a variety of vascular beds promoting tumor metastasis. Nat. Commun. 10, 18 (2019)

15. Overman, J. et al. R-propranolol is a small molecule inhibitor of the SOX18 transcription factor in a rare vascular syndrome and hemangioma. Elife 8 e43026 (2019).

16. Akiyama, H., Chaboissier, M. C., Martin, J. F., Schedl, A. \& de Crombrugghe, B. The transcription factor Sox 9 has essential roles in successive steps of the chondrocyte differentiation pathway and is required for expression of Sox 5 and Sox6. Genes Dev. 16, 2813-2828 (2002)

17. Barrionuevo, F. \& Scherer, G. SOX E genes: SOX 9 and SOX8 in mammalian testis development. Int. J. Biochem. Cell Biol. 42, 433-436 (2010).

18. Nowak, J. A., Polak, L., Pasolli, H. A. \& Fuchs, E. Hair follicle stem cells are specified and function in early skin morphogenesis. Cell Stem Cell 3, 33-43 (2008).

19. Scott, S. S. et al. Characterization of hemangioblastic traits within endothelial progenitor cells of multiple myeloma patients. Blood 116, 2984-2984 (2010).
20. Hanley, K. P. et al. Ectopic SOX9 mediates extracellular matrix deposition characteristic of organ fibrosis. J. Biol. Chem. 283, 14063-14071 (2008).

21. Lacraz, G. P. A. et al. Tomo-seq identifies SOX9 as a key regulator of cardiac fibrosis during ischemic injury. Circulation 136, 1396-1409 (2017).

22. Mack, J. J. \& Iruela-Arispe, M. L. NOTCH regulation of the endothelial cell phenotype. Curr. Opin. Hematol. 25, 212-218 (2018).

23. Chen, S. et al. Notch gain of function inhibits chondrocyte differentiation via Rbpj-dependent suppression of Sox9. J. Bone Min. Res. 28, 649-659 (2013).

24. Kalucka, J. et al. Single-cell transcriptome atlas of murine endothelial cells. Cell 180, 764-779 (2020). e720.

25. Patel, J. et al. Self-renewal and high proliferative colony forming capacity of late-outgrowth endothelial progenitors is regulated by cyclin-dependent kinase inhibitors driven by notch signaling. Stem Cells 34, 902-912 (2016).

26. Rinkevich, Y. et al. Skin fibrosis. Identification and isolation of a dermal lineage with intrinsic fibrogenic potential. Science 348, aaa2151 (2015).

27. Guo, W. et al. Slug and Sox 9 cooperatively determine the mammary stem cell state. Cell 148, 1015-1028 (2012).

28. Cooley, B. C. et al. TGF- $\beta$ signaling mediates endothelial-to-mesenchymal transition (EndMT) during vein graft remodeling. Sci. Transl. Med. 6, 227ra234-227ra234 (2014).

29. Evrard, S. M. et al. Endothelial to mesenchymal transition is common in atherosclerotic lesions and is associated with plaque instability. Nat. Commun. 7, 11853 (2016).

30. Whittam, A. J. et al. Challenges and opportunities in drug delivery for wound healing. Adv. Wound Care 5, 79-88 (2016).

31. Ingram, D. A. et al. Vessel wall-derived endothelial cells rapidly proliferate because they contain a complete hierarchy of endothelial progenitor cells. Blood 105, 2783-2786 (2005).

32. Naito, H., Kidoya, H., Sakimoto, S., Wakabayashi, T. \& Takakura, N. Identification and characterization of a resident vascular stem/progenitor cell population in preexisting blood vessels. EMBO J. 31, 842-855 (2012).

33. Schniedermann, J. et al. Mouse lung contains endothelial progenitors with high capacity to form blood and lymphatic vessels. BMC Cell Biol. 11, 50 (2010).

34. Crosby, C. O. et al. Quantifying the vasculogenic potential of induced pluripotent stem cell-derived endothelial progenitors in collagen hydrogels. Tissue Eng. Part A 25, 746-758 (2019).

35. Kusuma, S. et al. Self-organized vascular networks from human pluripotent stem cells in a synthetic matrix. Proc. Natl Acad. Sci. USA 110, 12601 (2013)

36. Stöckl, S., Göttl, C., Grifka, J. \& Grässel, S. Sox9 modulates proliferation and expression of osteogenic markers of adipose-derived stem cells (ASC). Cell. Physiol. Biochem. 31, 703-717 (2013).

37. Oh, C.-d et al. Identification of SOX9 interaction sites in the genome of chondrocytes. PLoS ONE 5, e10113 (2010).

38. Lamouille, S., Xu, J. \& Derynck, R. Molecular mechanisms of epithelial-mesenchymal transition. Nat. Rev. Mol. Cell Biol. 15, 178-196 (2014).

39. Kokudo, T. et al. Snail is required for TGFbeta-induced endothelialmesenchymal transition of embryonic stem cell-derived endothelial cells. J. Cell Sci. 121, 3317-3324 (2008).

40. Noseda, M. et al. Notch activation results in phenotypic and functional changes consistent with endothelial-to-mesenchymal transformation. Circ. Res. 94, 910-917 (2004).

41. Yung, L. M., Sánchez-Duffhues, G., Ten Dijke, P. \& Yu, P. B. Bone morphogenetic protein 6 and oxidized low-density lipoprotein synergistically recruit osteogenic differentiation in endothelial cells. Cardiovasc Res. 108, 278-287 (2015)

42. Leong, K. G. et al. Jagged1-mediated notch activation induces epithelial-tomesenchymal transition through slug-induced repression of E-cadherin. J. Exp. Med. 204, 2935-2948 (2007).

43. Niessen, K. et al. Slug is a direct Notch target required for initiation of cardiac cushion cellularization. J. Cell Biol. 182, 315-325 (2008).

44. Timmerman, L. A. et al. Notch promotes epithelial-mesenchymal transition during cardiac development and oncogenic transformation. Genes Dev. 18, 99-115 (2004).

45. Luanpitpong, S. et al. SLUG is required for SOX9 stabilization and functions to promote cancer stem cells and metastasis in human lung carcinoma. Oncogene 35, 2824-2833 (2016).

46. Kumar, S. et al. Sox9 activation highlights a cellular pathway of renal repair in the acutely injured mammalian kidney. Cell Rep. 12, 1325-1338 (2015).

47. Chakraborty, C., Sharma, A. R., Sharma, G., Doss, C. G. P. \& Lee, S. S. Therapeutic miRNA and siRNA: moving from bench to clinic as next generation medicine. Mol. Ther. Nucleic Acids 8, 132-143 (2017).

48. Liu, X. et al. Tumor-targeted in vivo gene silencing via systemic delivery of cRGD-conjugated siRNA. Nucleic Acids Res. 42, 11805-11817 (2014).

49. Huang, J. \& Guo, L. Knockdown of SOX9 inhibits the proliferation, invasion, and EMT in thyroid cancer cells. Oncol. Res. 25, 167-176 (2017).

50. Al Khateb, K. et al. In situ gelling systems based on Pluronic F127/Pluronic F68 formulations for ocular drug delivery. Int. J. Pharm. 502, 70-79 (2016). 
51. Kant, V. et al. Topical pluronic F-127 gel application enhances cutaneous wound healing in rats. Acta Histochem. 116, 5-13 (2014).

52. Plikus, M. V. et al. Epithelial stem cells and implications for wound repair. Semin. Cell Dev. Biol. 23, 946-953 (2012).

53. Pritchett, J., Athwal, V., Roberts, N., Hanley, N. A. \& Hanley, K. P. Understanding the role of SOX9 in acquired diseases: lessons from development. Trends Mol. Med. 17, 166-174 (2011).

54. Koh, C. M. in Methods in Enzymology Vol. 533 (ed. Lorsch, J.) Ch. 16 (Academic Press, 2013).

\section{Acknowledgements}

We would like to thank the flow cytometry facility of the Translational Research Institute for their assistance in the many cell sorting experiments undertaking.The study was funded by the Australian Research Council (ARC) Discovery Project (DP190103187). J.P. salary was supported by ARC Discovery Early Career Research Award (DE180100984). K.K. salary was supported by NHMRC Career Development Fellowship (APP1125290).

\section{Author contributions}

J.Z., J.P., M.F., M.C.Y., K.K.: acquisition of data, analysis and interpretation of data, obtained funding for the study and drafting of the manuscript. J.P., J.Z., I.H., S.K., H.H., J.D., H.Y.W., S-.L.S. and G.H.: acquisition of data and analysis. All authors have read the manuscript and approved it for publication.

\section{Competing interests}

J.P. and K.K. are co-inventors on a patent relating to the isolation of endothelial progenitors from the human placenta. The remaining authors declare no competing interests.

\section{Additional information}

Supplementary information The online version contains supplementary material available at https://doi.org/10.1038/s41467-021-22717-9.

Correspondence and requests for materials should be addressed to K.K.

Peer review information Nature Communications thanks the anonymous reviewer(s) for their contribution to the peer review of this work. Peer reviewer reports are available.

Reprints and permission information is available at http://www.nature.com/reprints

Publisher's note Springer Nature remains neutral with regard to jurisdictional claims in published maps and institutional affiliations.

(c) Open Access This article is licensed under a Creative Commons Attribution 4.0 International License, which permits use, sharing, adaptation, distribution and reproduction in any medium or format, as long as you give appropriate credit to the original author(s) and the source, provide a link to the Creative Commons license, and indicate if changes were made. The images or other third party material in this article are included in the article's Creative Commons license, unless indicated otherwise in a credit line to the material. If material is not included in the article's Creative Commons license and your intended use is not permitted by statutory regulation or exceeds the permitted use, you will need to obtain permission directly from the copyright holder. To view a copy of this license, visit http://creativecommons.org/ licenses/by/4.0/.

(C) The Author(s) 2021 